\title{
Pacific
}

Journal of

Mathematics

\section{BITWIST MANIFOLDS AND TWO-BRIDGE KNOTS}

James W. Cannon, William J. Floyd, LeeR Lambert, WALter R. Parry and Jessica S. PuRCell 


\title{
BITWIST MANIFOLDS AND TWO-BRIDGE KNOTS
}

\author{
James W. CANnon, William J. Floyd, LeeR Lambert, \\ WALTER R. PARRY AND JESSiCA S. PURCELL
}

\begin{abstract}
Though LeeR Lambert spent his life as an actuary and a musician and was a loving father of nine girls and one boy, he had always wanted to earn an advanced degree as a mathematician. With the encouragement of his wife, he earned his Ph.D. in mathematics at the age of 68. Many of the results of this paper appeared in his Ph.D. dissertation at Brigham Young University. At the age of 71, LeeR died of bone cancer. We miss you, LeeR.
\end{abstract}

We give uniform, explicit, and simple face-pairing descriptions of all the branched cyclic covers of the 3-sphere, branched over two-bridge knots. Our method is to use the bitwisted face-pairing constructions of Cannon, Floyd, and Parry; these examples show that the bitwist construction is often efficient and natural. Finally, we give applications to computations of fundamental groups and homology of these branched cyclic covers.

\section{Introduction}

Branched cyclic covers of $\mathbb{S}^{3}$ have played a major role in topology, and continue to appear in a wide variety of contexts. For example, branched cyclic covers of $\mathbb{S}^{3}$ branched over two-bridge knots have recently appeared in combinatorial work bounding the Matveev complexity of a 3-manifold [Petronio and Vesnin 2009], in algebraic and topological work determining relations between $L$-spaces, left-orderability, and taut foliations [Gordon and Lidman 2014; Boyer et al. 2013; Hu 2015], and in geometric work giving information on maps of character varieties [Nagasato and Yamaguchi 2012]. They provide a wealth of examples, and a useful collection of manifolds on which to study conjectures. Given their wide applicability, and their continued relevance, it is useful to have many explicit descriptions of these manifolds.

We give a new and elegant construction of the branched cyclic covers of twobridge knots, using the bitwist construction of [Cannon et al. 2009]. While other presentations of these manifolds are known (see, for example, [Minkus 1982; Mulazzani and Vesnin 2001]), we feel our descriptions have several advantages.

First, they follow from a recipe involving exactly the parameters necessary to describe a two-bridge knot, namely, continued fraction parameters. Our descriptions

MSC2010: 57M12, 57M25.

Keywords: bitwist manifolds, two-bridge knots, branched cyclic covers. 
apply uniformly to all two-bridge knots, and all branched cyclic covers of $\mathbb{S}^{3}$ branched over two-bridge knots.

Second, they are obtained from a description of a two-bridge knot using a very straightforward bitwisted face-pairing construction, as in [Cannon et al. 2000; 2002; 2003; 2009]. Bitwisted face-pairings (read "bi-twisted", as in twisted two ways) are known to produce all closed orientable 3-manifolds. The examples of this paper show, in addition, that bitwist constructions are often efficient and natural. While a generic face-pairing will yield a pseudomanifold, which, with probability 1, will not be an actual manifold [Dunfield and Thurston 2006], bitwisted face-pairings avoid this problem. (We will review necessary information on bitwisted face-pairings, so no prior specialized knowledge is required to understand our constructions.)

Third, our description leads to immediate consequences in geometric group theory. We obtain a simple proof of the fact that the fundamental group of the $n$-fold branched cyclic covering of $\mathbb{S}^{3}$, branched over a two-bridge knot, has a cyclic presentation. Our description also gives immediate presentations of two well-known families of groups, the Fibonacci and Sieradski groups. These are known to arise as fundamental groups of branched cyclic covers of $\mathbb{S}^{3}$ branched over the figure-eight and trefoil knots, respectively. These groups have received considerable attention from geometric group theorists; see, for example, [Cavicchioli et al. 1998] for further references, and Section 6 for more history. Our methods recover the fact that the first homology groups of Sieradski manifolds are periodic. We also give a proof that their fundamental groups are distinct using Milnor's characterization of these spaces. We consider orders of abelianizations of Fibonacci groups as well. These orders form an interesting sequence related to the Fibonacci sequence, which we shall see.

1A. Bitwisted face-pairing description. We will see that the bitwist description of any two-bridge knot is encoded as the image of the north-south axis in a ball labeled as in Figure 1, along with an associated vector of integer multipliers. For the branched cyclic cover, the description is encoded by adding additional longitudinal arcs to the sphere. We now describe the construction briefly, in order to state the main results of the paper. A more detailed description of the construction, with examples, is given in Section 2.

Begin with a finite graph $\Gamma$ in the 2-sphere $\mathbb{S}^{2}=\partial \mathbb{B}^{3}$ that is the union of the equator $e$, one longitude $N S$ from the north pole $N$ to the south pole $S$, and $2 k \geq 0$ latitudinal circles, such that $\Gamma$ is invariant under reflection

$$
\epsilon: \mathbb{S}^{2} \rightarrow \mathbb{S}^{2}
$$

in the equator. Then $\Gamma$ divides $\mathbb{S}^{2}$ into $2(k+1)$ faces that are paired by $\epsilon$. This face-pairing is shown in Figure 1. 


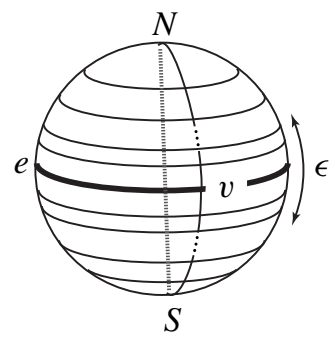

Figure 1. The model face-pairing: a faceted 3-ball with dotted central axis and reflection face-pairing $\epsilon: \partial \mathbb{B}^{3} \rightarrow \partial \mathbb{B}^{3}$.

As with any face-pairing, the edges fall into edge cycles. The equator $e$ forms one edge cycle $c_{0}$ since the reflection $\epsilon$ leaves $e$ invariant. Each other edge of the graph is matched with its reflection to form another edge cycle $c_{i}$. We number these edge cycles from 0 through $2 k+1$, with even numbers associated with latitudinal edges, as indicated in Figure 2.

Now choose nonzero integer multipliers, denoted $m_{0}, m_{1}, \ldots, m_{2 k}, m_{2 k+1}$, for the edge cycles $c_{i}$. In the case at hand, restrict the choice of multipliers $m_{i}$ as follows. Each latitudinal edge cycle $c_{2 i}$ is assigned either +1 or -1 as multiplier. Each longitudinal edge cycle $c_{2 i+1}$ may be assigned any integer multiplier $m_{2 i+1}$ whatsoever, including 0 . The multiplier $m_{2 i+1}=0$ is usually forbidden, but in this case indicates that the two edges of edge-class $c_{2 i+1}$ must be collapsed to a point before the bitwist construction is engaged.

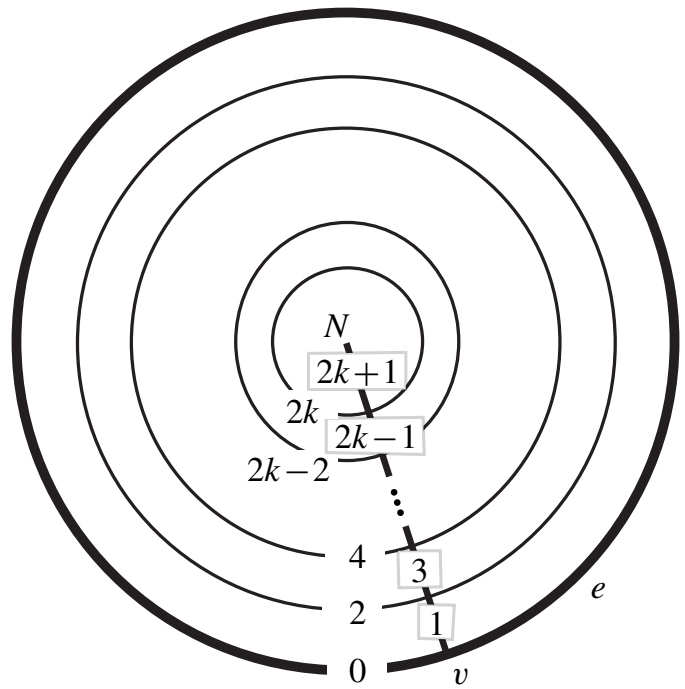

Figure 2. The northern hemisphere, with edge cycles numbered. 
Finally, for the general bitwist construction, we obtain a closed manifold $M(\epsilon, m)$ by taking the following quotient. First, subdivide each edge in the edge cycle $c_{i}$ into $\left|c_{i}\right| \cdot\left|m_{i}\right|$ subedges. Insert an additional edge between each adjacent positive and negative edge, if any. Then twist each subedge by one subedge in a direction indicated by the sign of $m_{i}$. Finally, apply the face-pairing map $\epsilon$ to glue bitwisted faces. This is the bitwist construction.

In Theorem 4.2, we prove that the bitwist manifold $M(\epsilon, m)$ described above is the 3-sphere $\mathbb{S}^{3}$. The image of the north-south axis in $\mathbb{S}^{3}$ is a two-bridge knot. In fact, we prove more. Recall that every two-bridge knot is the closure of a rational tangle. See [Kauffman and Lambropoulou 2002] for an elementary exposition. A rational tangle is determined up to isotopy by a single rational number, which we call the rational number invariant of the tangle. There are two natural ways to close a tangle so that it becomes a knot or link, the numerator closure and the denominator closure. The full statement of Theorem 4.2 is below.

Theorem 4.2. The bitwist manifold $M(\epsilon, m)$ is the 3-sphere $\mathbb{S}^{3}$. The image of the north-south axis in $\mathbb{S}^{3}$ is the two-bridge knot which is the numerator closure of the tangle $T(a / b)$ whose rational number invariant $a / b$ is

$$
2 \cdot m_{0}+\frac{1}{2 \cdot m_{1}+\frac{1}{\ddots \cdot+\frac{1}{2 \cdot m_{2 k}+\frac{1}{2 \cdot m_{2 k+1}}}}} .
$$

Remark 1.1. The 2's in the continued fraction indicate that the tangle is constructed using only full twists instead of the possible mixture of full and half twists.

Example 1.2. The simplest case, with only equator and longitude, yields the trefoil and figure-eight knots, as we shall see in Theorem 4.1. Simple subdivisions yield their branched cyclic covers, the Sieradski [1986] and Fibonacci [Vesnin and Mednykh 1996] manifolds.

Definition 1.3. We say that the multiplier function $m$ is normalized if

(1) $m_{2 k+1} \neq 0$, and

(2) if $m_{2 i+1}=0$ for some $i \in\{0, \ldots, k-1\}$, then $m_{2 i}=m_{2 i+2}$.

With this definition, the previous theorem and well-known results involving two-bridge knots yield the following corollary.

Corollary 4.4. Every normalized multiplier function yields a nontrivial two-bridge knot. Conversely, every nontrivial two-bridge knot $K$ is realized by either one or two normalized multiplier functions. If $K$ is the numerator closure of the tangle $T(a / b)$, then it has exactly one such realization if and only if $b^{2} \equiv 1 \bmod a$. 
Notice that the $n$-th branched cyclic covering of $\mathbb{S}^{3}$, branched over $K$, can be obtained by unwinding the description $n$ times about the unknotted axis that represents $K$, unwinding the initial face-pairing as in Figure 17. This leads to a new proof of the following result, originally due to Alberto Cavicchioli, Friedrich Hegenbarth and Ana Chi Kim [Cavicchioli et al. 1999a].

Theorem 5.2. The fundamental group of the $n$-th branched cyclic covering of $\mathbb{S}^{3}$, branched over a two-bridge knot $K$, has a cyclic presentation.

Problem 1.4. How should one carry out the analogous construction for arbitrary knots?

1B. The Fibonacci and Sieradski manifolds. Since the knots in the face-pairing description appear as the unknotted axis in $\mathbb{B}^{3}$, it is easy to unwind $\mathbb{B}^{3}$ around the axis to obtain face-pairings for the branched cyclic coverings of $\mathbb{S}^{3}$, branched over the trefoil knot and the figure-eight knot. For the trefoil knot, the $n$-th branched cyclic cover $S_{n}$ is called the $n$-th Sieradski manifold. For the figure-eight knot, the $n$-th branched cyclic cover $F_{n}$ is called the $n$-th Fibonacci manifold. We will prove:

Theorem 5.4. The fundamental group $\pi_{1}\left(F_{n}\right)$ is the $2 n$-th Fibonacci group with presentation

$$
\left\langle x_{1}, \ldots, x_{2 n} \mid x_{1} x_{2}=x_{3}, x_{2} x_{3}=x_{4}, \ldots, x_{2 n-1} x_{2 n}=x_{1}, x_{2 n} x_{1}=x_{2}\right\rangle .
$$

The fundamental group $\pi_{1}\left(S_{n}\right)$ is the $n$-th Sieradski group with presentation

$$
\left\langle y_{1}, \ldots, y_{n} \mid y_{1}=y_{2} y_{n}, y_{2}=y_{3} y_{1}, y_{3}=y_{4} y_{2}, \ldots, y_{n}=y_{1} y_{n-1}\right\rangle \text {. }
$$

Remark 1.5. The group presentations are well known once the manifolds are recognized as branched cyclic covers of $\mathbb{S}^{3}$, branched over the figure-eight knot and the trefoil knot. But these group presentations also follow immediately from the description of the bitwist face-pairings, as we shall see.

The first homology of the Sieradski manifolds has an intriguing periodicity property, which is well known (see, for example, Rolfsen [1976]). In particular, it is periodic of period 6 . The following theorem, concerning their fundamental groups, is not as well known; it is difficult to find in the literature. We give a proof using Milnor's characterization of these spaces.

Theorem 5.13. No two of the Sieradski groups are isomorphic. Hence no two of the branched cyclic covers of $\mathbb{S}^{3}$, branched over the trefoil knot, are homeomorphic.

1C. Organization. In Section 2, we give a more careful description of the bitwisted face-pairing, and work through the description for two examples, which will correspond to the trefoil and figure-eight knots. 
In Section 3, we recall many of the results in our previous papers [Cannon et al. 2003; 2009] to make explicit the connections between face-pairings, Heegaard splittings, and surgery descriptions of 3-manifolds. We apply these to the examples of bitwisted face-pairings given here, to give surgery descriptions. We use these descriptions in Section 4 to prove that our constructions yield two-bridge knots. The proofs of the main geometric theorems are given in this section.

In Section 5 we turn to geometric group theory. We prove that our presentations easily lead to well-known results on presentations of fundamental groups. We also give results on Fibonacci and Sieradski groups in this section.

Finally, Section 6 explains some of the history of these problems.

\section{Bitwisted face-pairing: trefoil and figure-eight knots}

In this section we step through the bitwisted face-pairing description more carefully. We believe it will be most useful to work through a pair of examples. We will see in subsequent sections that these examples lead to Fibonacci and Sieradski manifolds.

As an example, consider the simplest model, shown in Figure 3 (left). The graph has three edges and three vertices, and divides the sphere into two singular "triangles", which are then matched by reflection $\epsilon$ in the equator $e$.

Bitwisted face-pairings require an integer multiplier for each edge cycle. For this simple model there are two edge cycles, namely the singleton $c_{0}=\{e\}$ and the pair $c_{1}=\{N v, S v\}$. We will see that multiplying every multiplier by -1 takes the knot which we construct to its mirror image. So up to taking mirror images, the two simplest choices for multipliers are $m_{0}= \pm 1$ for $c_{0}$ and $m_{1}=1$ for $c_{1}$. The bitwist construction requires that each edge in the cycle $c_{i}$ be subdivided into $\left|c_{i}\right| \cdot\left|m_{i}\right|$ subedges. When both positive and negative multipliers appear on edges of the same face, we must insert an additional edge, called a sticker, between a negative
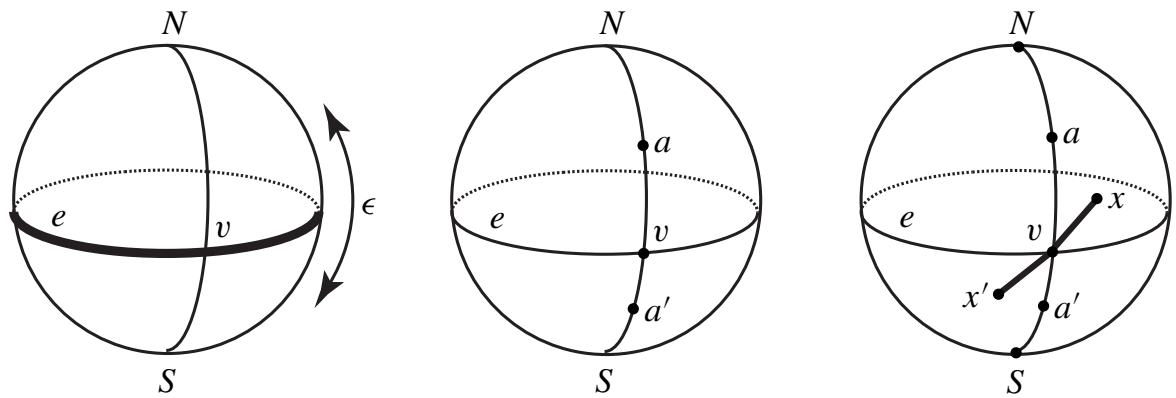

Figure 3. A faceted 3-ball with vertices $N$, $v$, and $S$ and edges $N v$, $S v$, and $e$ (left), subdivisions for $M_{+}$(middle), and subdivisions for $M_{-}$(right). 
and positive edge in a given, fixed orientation of $\mathbb{S}^{2}$. We will use the clockwise orientation.

With the facets modified as described in the previous paragraph, we are prepared for the bitwisting. Twist each subedge of each face by one subedge before applying the model map $\epsilon$. Edges with positive multiplier are twisted in the direction of the fixed orientation. Edges with negative multiplier are twisted in the opposite direction. The stickers resolve the twisting conflict between negative and positive subedges. A sticker in the domain of the map splits into two subedges. A sticker in the range of the map absorbs the folding together of two subedges.

We denote by $M_{+}$the face-pairing in which both multipliers are +1 and by $M_{-}$ the face-pairing where one multiplier is +1 and the other is -1 . The two results are shown in Figure 3 (middle and right).

After this subdivision, the faces can be considered to have five edges for $M_{+}$ and seven edges for $M_{-}$. Before making the identification of the northern face with the southern face, we rotate the 5-gon one notch (= one edge $=$ one-fifth of a turn, combinatorially) in the direction of the given orientation on $\mathbb{S}^{2}$ before identification. We rotate the edges of the 7 -gon with positive multiplier one notch $(=$ one edge $=$ one-seventh of a turn, combinatorially) in the direction of the orientation before identification. The edges with negative multiplier are twisted one notch in the opposite direction. The stickers absorb the conflict at the joint between positive and negative. Thus the face-pairings $\epsilon_{+}$and $\epsilon_{-}$in terms of the edges forming the boundaries of the faces are given as follows.

For $M_{+}$:

$$
\epsilon_{+}:\left(\begin{array}{ccccc}
a v & e & v a & a N & N a \\
e & v a^{\prime} & a^{\prime} S & S a^{\prime} & a^{\prime} v
\end{array}\right)
$$

For $M_{-}$:

$$
\epsilon_{-}:\left(\begin{array}{ccccccc}
a v & e & v x & x v & v a & a N & N a \\
v x^{\prime} & x^{\prime} v & e & v a^{\prime} & a^{\prime} S & S a^{\prime} & a^{\prime} v
\end{array}\right)
$$

The bitwist theorem [Cannon et al. 2009, Theorem 3.1] implies that the resulting identification spaces are closed manifolds, which we denote by $F_{1}$ for $M_{+}$and $S_{1}$ for $M_{-}$. We shall see that both of these manifolds are $\mathbb{S}^{3}$, and thus topologically uninteresting. But as face-pairings, these identifications are wonderfully interesting because the north-south axis from $\mathbb{B}^{3}$ becomes the figure-eight knot $K_{+}$in $F_{1}$ and becomes the trefoil knot $K_{-}$in $S_{1}$. We prove this in Theorem 4.1.

\section{Pseudo-Heegaard splittings and surgery diagrams}

In order to recognize the quotients of $\mathbb{B}^{3}$ described in Section 1 as the 3-sphere and to recognize the images of the north-south axis as two-bridge knots, we need to make more explicit the connections between face-pairings, Heegaard splittings, and surgery descriptions of 3-manifolds, described in our previous papers [Cannon 

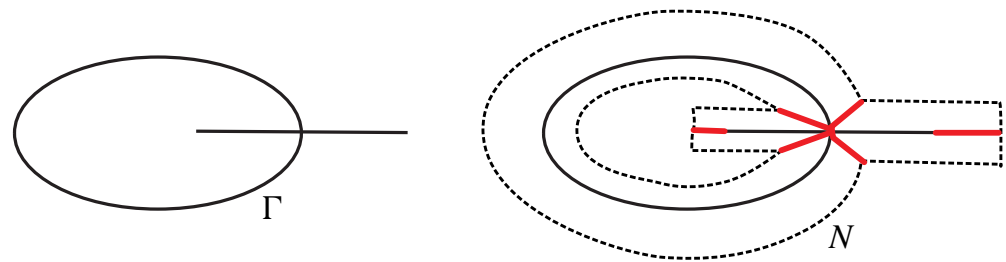

Figure 4. The addition of new red arcs.

et al. 2003; 2009]. We use these connections to transfer knots from the face-pairing description to the surgery descriptions.

3A. The pseudo-Heegaard splitting. We begin with the following information:

$B$ : a faceted 3-ball which we identify with $\mathbb{B}^{3}=[0,1] \cdot \mathbb{S}^{2}$ (where $\cdot$ is scalar multiplication).

$\Gamma \subset \partial B=\mathbb{S}^{2}$ : the 1 -skeleton of $B$, a connected, finite graph with at least one edge.

$\Delta$ : the dual 1-skeleton, consisting of a cone from the center 0 of $B$ to points of $\partial B$, one in the interior of each face of $B$.

$N$ : a regular neighborhood of $\Gamma$ in $\partial B$.

$N_{\Gamma}=\left[\frac{3}{4}, 1\right] \cdot N$ : a regular neighborhood of $\Gamma$ in $B$.

$N_{\Delta}=\operatorname{cl}\left(B-N_{\Gamma}\right)$ : a regular neighborhood of $\Delta$ in $B$.

Add extra structure to $N$ and $N_{\Gamma}$ as follows.

First, from each vertex $v$ of $\Gamma$, we extend arcs from $v$ to $\partial N$, one to each local side of $\Gamma$ at $v$ so that the interiors of these arcs are mutually disjoint. Label these arcs red. Figure 4 shows this for the simplest model described above.

Next, momentarily disregarding both the vertices and edges of $\Gamma$, we view the red arcs as subdividing $N$ into quadrilaterals (occasionally singular at the arc ends), every quadrilateral having two sides in $\partial N$ and two sides each of which is the union of two (or one in the singular case) of these red arcs, as on the left of Figure 5.

Every such quadrilateral contains exactly one edge of $\Gamma$. We cut these quadrilaterals into half-quadrilaterals by arcs transverse to the corresponding edge of $\Gamma$ at the middle of that edge. Label these transverse arcs blue. For the simplest model, this is shown in Figure 5 (right).

If we cut $N$ along the new red arcs and blue transverse arcs, multiply by the scalar interval $\left[\frac{3}{4}, 1\right]$, and desingularize, we obtain cubes, each containing exactly one vertex of $\Gamma$ in its boundary. Endow these cubes with a cone structure, coned to its vertex in $\Gamma$. See Figure 6.

Finally, we assume that $\epsilon: \partial B \rightarrow \partial B$ is an orientation-reversing face-pairing, based on the faceted 3-ball $B$, that respects all of this structure as much as possible: 

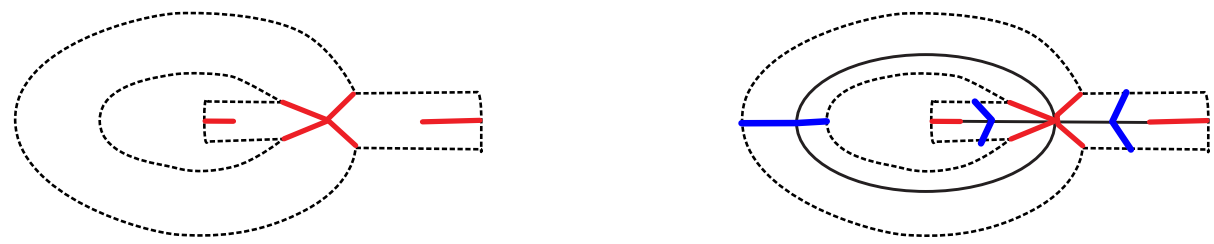

Figure 5. The addition of blue transverse arcs.

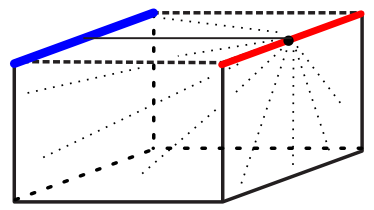

Figure 6. The cone structure.

faces are paired; $N$ is invariant under the pairing; the regions bounded by the new arcs, the transverse arcs, the boundary of $N$, and $\Gamma$ are paired by $\epsilon$; and cone structures are preserved.

Definition 3.1. Let $C_{\Gamma}$ be the union of the products of the transverse arcs with $\left[\frac{3}{4}, 1\right]$. Let $C_{\Delta}=N_{\Delta} \cap(\partial B)$. Define $D_{\Gamma}=C_{\Gamma} / \epsilon, D_{\Delta}=C_{\Delta} / \epsilon, H_{\Gamma}=N_{\Gamma} / \epsilon$, and $H_{\Delta}=N_{\Delta} / \epsilon$, and let $\delta=\partial D_{\Delta}$ and $\gamma=\partial D_{\Gamma}$.

The following is essentially contained in [Cannon et al. 2003, Theorem 4.2.1].

Theorem 3.2. The space $H_{\Delta}$ is a handlebody with one handle for each face pair of $B$. The set $D_{\Delta}$ is a disjoint union of disks that form a complete set of handle disks for $H_{\Delta}$; the curves $\delta$ form a complete set of handle curves.

The space $H_{\Gamma}$ is a handlebody if and only if $M(\epsilon)=B / \epsilon$ is a 3-manifold. In that case, $D_{\Gamma}$ is a disjoint union of disks that form a complete set of handle disks for $H_{\Gamma}$ and $\gamma$ forms a complete set of handle curves. Whether $M(\epsilon)$ is a manifold or not, the disks of $D_{\Gamma}$ cut $H_{\Gamma}$ into pieces $X_{i}$, each containing exactly one vertex $v_{i}$ of $M(\epsilon)$, and each $X_{i}$ is a cone $v_{i} S_{i}$, where $S_{i}$ is a closed orientable surface. The space $M(\epsilon)$ is a manifold if and only if each $S_{i}$ is a 2-sphere. (The cone structure on $X_{i}$ uses the cone structures of the pieces described above.)

Terminology 3.3. Even when $M(\epsilon)=B / \epsilon$ is not a manifold, we call the disks of $D_{\Gamma}$ handle disks for $H_{\Gamma}$ and the curves $\gamma=\partial D_{\Gamma}$ handle curves for $H_{\Gamma}$. We call $H_{\Gamma}$ a pseudohandlebody and the pair $\left(H_{\Gamma}, H_{\Delta}\right)$ a pseudo-Heegaard splitting for $M(\epsilon)$.

All bitwist manifolds based on the face-pairing $(B, \epsilon)$ have Heegaard splittings and surgery descriptions that can be based on any unknotted embedding of $H_{\Delta}=$ $N_{\Delta} / \epsilon$ in $\mathbb{S}^{3}=\mathbb{R}^{3} \cup\{\infty\}$. The closure of the complement is then also a handlebody, which we shall denote by $H$. We describe here a particular unknotted embedding 

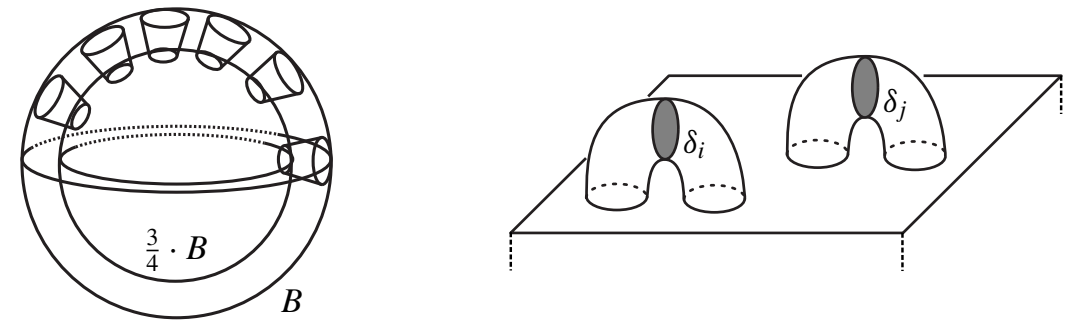

Figure 7. The ball with chimneys $N_{\Delta}$ (left), and the handlebody $H_{\Delta}$ (right).

of $H_{\Delta}$ in $\mathbb{S}^{3}$, and illustrate with the constructions from Section $1 \mathrm{~A}$, especially those of Section 2.

Note that $N_{\Delta}=\left(\left[0, \frac{3}{4}\right] \cdot \mathbb{S}^{2}\right) \cup\left(\left[\frac{3}{4}, 1\right] \cdot C_{\Delta}\right)$, where $\left[0, \frac{3}{4}\right] \cdot \mathbb{S}^{2}$ is, of course, a 3-ball, and $\left[\frac{3}{4}, 1\right] \cdot C_{\Delta}$ is a family of chimneys attached to that 3 -ball, as in Figure 7 (left).

The space $H_{\Delta}$ is formed by identifying the tops of those chimneys in pairs. We may therefore assume $H_{\Delta}$ is embedded in $\mathbb{S}^{3}=\mathbb{R}^{3} \cup\{\infty\}$ as shown in Figure 7 (right). We identify $\left[0, \frac{3}{4}\right) \cdot \mathbb{S}^{2}$ with $\mathbb{R}^{2} \times(-\infty, 0) \subset \mathbb{R}^{3}$. The 2 -sphere $\left(\frac{3}{4}\right) \cdot \mathbb{S}^{2}$ minus one point is identified with $\mathbb{R}^{2} \times\{0\} \subset \mathbb{R}^{3}$. The chimneys with tops identified become handles.

3B. Pseudo-Heegaard splittings of our examples. For the constructions of Sections $1 \mathrm{~A}$ and 2, we now determine the curves $\delta$ and $\gamma$ on the handlebody $H_{\Delta}$.

Begin with the simple face-pairing description of Section 2. The handlebody $H_{\Delta}$ is embedded in $\mathbb{R}^{3} \cup\{\infty\}$ as above, with the plane $\mathbb{R}^{2} \times\{0\}$ identified with $\left(\frac{3}{4}\right) \cdot \mathbb{S}^{3}$ minus a point. Sketch the graph $\left(\frac{3}{4}\right) \cdot \Gamma$ on $\mathbb{R}^{2} \times\{0\}$, with the vertex $\left(\frac{3}{4}\right) \cdot v$ at $\infty$, as in Figure 8 (left). There is just one pair of faces, hence just one handle in this case, as shown. Thus $D_{\Delta}$ is a single disk with boundary $\delta$, shown in the left side of the figure.

We need to determine the curves $\gamma=\partial D_{\Gamma}$. Recall that $D_{\Gamma}=C_{\Gamma} / \epsilon$, and the disks $C_{\Gamma}$ consist of the union of the products of the blue transverse arcs with $\left[\frac{3}{4}, 1\right]$.
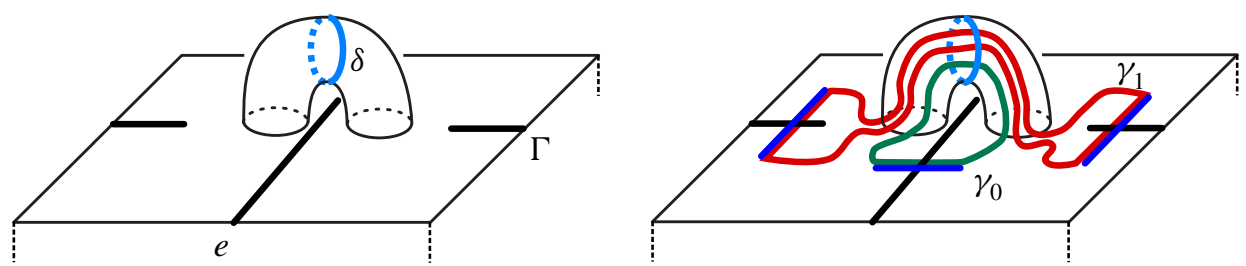

Figure 8. The graph $\left(\frac{3}{4}\right) \cdot \Gamma$ and curve $\delta$ for the simple example (left), and the graph with curves $\gamma$ added in, running partly along blue transverse arcs (right). 
Thus curves in $\gamma$ will contain blue transverse arcs, as well as arcs along the handles of $H_{\Delta}$, running from the blue transverse arcs to a curve $\delta_{j}$.

In the case of the simple example, following the action of $\epsilon$, we see that the transverse arc $\tau_{0}$ dual to the edge $e$ gives a single simple closed curve $\gamma_{0}$ that follows $\tau_{0}$, then connects the endpoints of $\tau_{0}$ via an arc that runs over the single handle of $H_{\Delta}$. The two transverse arcs dual to $N v$ and $S v$ are identified by $\epsilon$. Thus endpoints of these arcs are connected by arcs running over the handle. We obtain a simple closed curve $\gamma_{1}$. This is shown in Figure 8 (right).

The general picture, for the construction of Section 1A, follows similarly. We summarize in a lemma.

Lemma 3.4. Let $\Gamma$ and $\epsilon$ be as in Section 1A, with $\Gamma$ the union of the equator $e$, one longitude $N S$ from the north pole $N$ to the south pole $S$, and $2 k \geq 0$ latitudinal circles, such that $\Gamma$ is invariant under reflection $\epsilon$ in the equator. Then the handle curves on $H_{\Delta}$ for this face-pairing are as follows.

(1) There are $k+1$ handles of $H_{\Delta}$, corresponding to the $k+1$ regions in the complement of $\Gamma$ in the northern hemisphere, each running from the region to its mirror region in the southern hemisphere. These give curves $\delta_{0}, \ldots, \delta_{k}$ encircling the handles.

(2) The transverse arc dual to the edge e gives a curve $\gamma_{0}$ with endpoints connecting to itself over the handle corresponding to the faces on either side of e, which are identified by $\epsilon$.

(3) Each latitudinal arc distinct from e, if any, is joined to its mirror over two handles, one for each face on opposite sides of the latitudinal edge. These give curves $\gamma_{2 i}, i=1, \ldots, k$, with index corresponding to the edge label as in Figure 2.

(4) Each transverse arc dual to a longitudinal arc is joined to its mirror over a handle corresponding to the region on either side of that arc. These give curves $\gamma_{2 i+1}, i=0, \ldots, k$, with index corresponding to edge label as in Figure 2.

Curves parallel to those of Lemma 3.4 are illustrated in Figure 9. These curves have been pushed slightly to be disjoint, in a manner described in the next section.

3C. The surgery description. We assume now that we are given a bitwist construction based on $(B, \epsilon)$. We are given the following information:

$c_{1}, \ldots, c_{k}$ : the edge cycles of $\epsilon$.

$m=\left\{m_{1}, \ldots, m_{k}\right\}$ : a set of nonzero integer multipliers assigned to these edge cycles.

$\epsilon_{m}: \partial B \rightarrow \partial B:$ the associated bitwist face-pairing.

$M(\epsilon, m)=M\left(\epsilon_{m}\right)=B / \epsilon_{m}:$ the resulting bitwist manifold. 


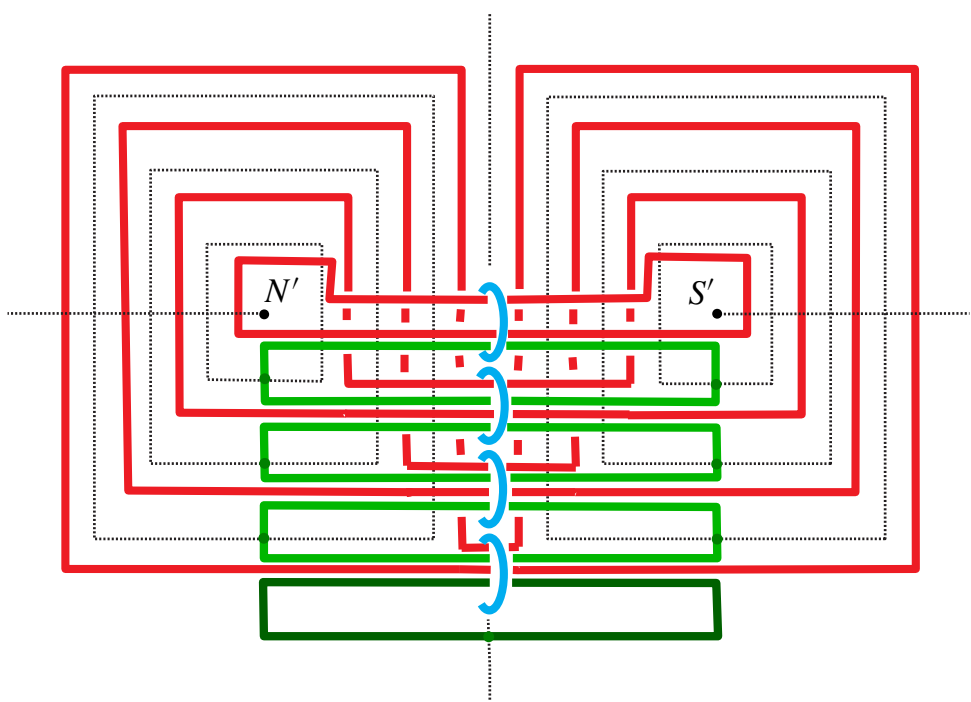

Figure 9. The curves $\delta$ and $\gamma$ in $H_{\Delta}$. Curves $\delta$ are shown in blue, $\gamma_{0}$ is in dark green at the bottom of the diagram, curves of $\gamma$ corresponding to latitudinal transverse arcs are in green, and curves of $\gamma$ corresponding to longitudinal transverse arcs are in red.

The set $\delta=\partial D_{\Delta}$ is a disjoint union of simple closed handle curves $\delta_{1}, \ldots, \delta_{g}$ for $H_{\Delta}$, one for each face pair of $\epsilon$. We first push each $\delta_{i}$ slightly into $\mathbb{R}^{3} \backslash H_{\Delta}$ to a curve $\delta_{i}^{\prime}$. We let $V_{i}$ denote a solid torus neighborhood of $\delta_{i}^{\prime}$ in $\mathbb{R}^{3} \backslash H_{\Delta}$, remove it, and sew a new solid torus $V_{i}^{\prime}$ back in with meridian and longitude reversed (0-surgery on each $\left.\delta_{i}^{\prime}\right)$. The curve $\delta_{i}$ now bounds a disk $E_{i}$, disjoint from $H_{\Delta}$, consisting of an annulus from $\delta_{i}$ to $\partial V_{i}^{\prime}$ and a meridional disk in $V_{i}^{\prime}$. The result is a new handlebody

$$
H^{\prime}=\left[\operatorname{cl}\left(\mathbb{S}^{3} \backslash H_{\Delta}\right) \backslash \bigcup V_{i}\right] \cup\left[\cup V_{i}^{\prime}\right]
$$

with the same handle curves $\delta_{1}, \ldots, \delta_{g}$ as $H_{\Delta}$ and with handle disks $E_{1}, \ldots, E_{g}$. The union $H_{\Delta} \cup H^{\prime}$ is homeomorphic to $\left(\mathbb{S}^{2} \times \mathbb{S}^{1}\right) \# \ldots \#\left(\mathbb{S}^{2} \times \mathbb{S}^{1}\right)$.

The set $\gamma=\partial D_{\Gamma}$ is a disjoint union of simple closed curves $\gamma_{1}, \ldots, \gamma_{k}$ on $\partial H^{\prime}$, one for each edge class of $\epsilon$. We push each $\gamma_{j}$ slightly into $\operatorname{int}\left(H^{\prime}\right) \backslash\left(\bigcup V_{i}^{\prime}\right)$ to a curve $\gamma_{j}^{\prime}$. On each $\gamma_{j}^{\prime}$ we perform $\operatorname{lk}\left(\gamma_{j}, \gamma_{j}^{\prime}\right)+\left(1 / m_{j}\right)$ surgery. Note from Lemma 3.4 that in our applications, the curves $\gamma_{j}$ will be unknotted and the curves $\gamma_{j}^{\prime}$ will have linking number 0 with them.

These surgeries modify $H^{\prime}$ to form a new handlebody $H^{\prime \prime}$. By [Cannon et al. 2009, Theorem 4.3], $\left(H_{\Delta}, H^{\prime \prime}\right)$ is a Heegaard splitting for $M(\epsilon, m)$ (or, because of ambiguities associated with orientations, the manifold $M(\epsilon,-m)$, with $-m=$ $\left\{-m_{1}, \ldots,-m_{k}\right\}$, which is homeomorphic with $\left.M(\epsilon, m)\right)$. 
For our purposes, it is important to see that these surgeries can be realized by an explicit homeomorphism from $H^{\prime}$ to $H^{\prime \prime}$ defined by Dehn-Lickorish moves. To that end, we enclose $\gamma_{j}^{\prime}$ in a solid torus neighborhood $U_{j}$ that is joined to $\gamma_{j}$ by an annulus $A_{j}$. We remove $U_{j}$ and cut the remaining set along $A_{j}$. Let $A_{j}^{\prime}$ denote one side of the cut. We may parametrize a neighborhood of $A_{j}^{\prime}$ by $(\theta, s, t)$, where $\theta \in \mathbb{R}(\bmod 2 \pi)$ is the angle around the circle $\gamma_{j}, s \in[0,1]$ is the depth into $H^{\prime}$, and $t \in[0,1]$ is the distance from $A_{j}^{\prime}$. Then one twists this neighborhood of $A_{j}^{\prime}$ by the map $(\theta, s, t) \mapsto\left(\theta+(1-t) \cdot m_{j} \cdot 2 \pi, s, t\right)$ before reattaching $A_{j}^{\prime}$ to its partner $A_{j}^{\prime \prime}$ to reconstitute $A_{j}$. This twisting operation defines a homeomorphism $\phi:\left[H^{\prime} \backslash\left(\bigcup U_{j}\right)\right] \rightarrow\left[H^{\prime} \backslash\left(\bigcup U_{j}\right)\right]$. One then reattaches the solid tori $U_{j}$ via the homeomorphisms $\left.\phi\right|_{\partial U_{j}}$ to form $H^{\prime \prime}$, with an extended homeomorphism $\Phi: H^{\prime} \rightarrow H^{\prime \prime}$. The homeomorphism $\Phi$ is the identity except in a small neighborhood of $\gamma$. The new handle disks are $\Phi\left(E_{1}\right), \ldots, \Phi\left(E_{g}\right)$.

We apply this to obtain a surgery description for our construction. Recall from Section $1 \mathrm{~A}$ that our multipliers were chosen to be \pm 1 on latitudinal edge cycles, and any integer $m_{i}$ on longitudinal edges. We record the result in the following lemma.

Lemma 3.5. Let $\Gamma$ and $\epsilon$ be as in Section 1A, with handle curves as in Lemma 3.4. Then the manifold $M(\epsilon, m)$ has the following surgery description.

(1) There are $k+1$ simple closed curves $\delta_{0}^{\prime}, \ldots, \delta_{k}^{\prime}$, with each $\delta_{j}^{\prime}$ parallel to $\delta_{j}$, pushed to the exterior of the handle of $H_{\Delta}$. Each $\delta_{j}^{\prime}$ has surgery coefficient 0.

(2) Each curve of $\gamma$ corresponding to a latitudinal edge class $\gamma_{2 i}$ appears with surgery coefficient $m_{2 i}= \pm 1, i=0, \ldots, k$.

(3) Each curve of $\gamma$ corresponding to longitudinal edge class $\gamma_{2 i+1}$ has surgery coefficient $1 / m_{2 i+1}$. If one of these multipliers is 0 , so that the edge collapses to a point and disappears as an edge class, we retain the corresponding curve, but with surgery coefficient $\frac{1}{0}=\infty$.

The curves are shown in Figure 9.

3D. The knot as the image of the north-south axis. It is now an easy matter to identify the image of the north-south axis in our bitwist constructions. In particular, we want to recognize this curve in the associated surgery description of the manifold. The portion of the curve in the handlebody $H_{\Delta}$ is obvious. That portion in the handlebody $H_{\Gamma}$ is simple, yet not so obvious. We need a criterion that allows us to recognize it.

To that end, suppose that $H_{\Gamma}$ is a pseudohandlebody with one vertex $x$. Recall that $H_{\Gamma} \backslash D_{\Gamma}$ has a natural cone structure from $x$. We say that an $\operatorname{arc} \alpha$ in $H_{\Gamma} \backslash D_{\Gamma}$ is boundary parallel if there is a disk $D$ in $H_{\Gamma} \backslash D_{\Gamma}$ such that $(\partial D) \cap\left(\operatorname{int}\left(H_{\Gamma}\right)\right)=\operatorname{int}(\alpha)$ and $(\partial D) \cap\left(\partial H_{\Gamma}\right)$ is an $\operatorname{arc} \alpha^{\prime}$. 
Lemma 3.6. Suppose $a, b \in\left(\partial H_{\Gamma}\right) \backslash D_{\Gamma}$ with $a \neq b$. Then the arc $\alpha=a x \cup b x$ (using the cone structure) is boundary parallel, and any arc $\beta$ that has $a$ and $b$ as endpoints and is boundary parallel is, in fact, isotopic to $\alpha$.

Proof. The set $D_{\Gamma}$ is a disjoint union of handle disks for $H_{\Gamma}$, hence does not separate $\partial H_{\Gamma}$. There is therefore an arc $\alpha^{\prime}$ from $a$ to $b$ in $\left(\partial H_{\Gamma}\right) \backslash D_{\Gamma}$. The disk $x \alpha^{\prime}$, which uses the cone structure, proves that $\alpha$ is boundary parallel. If $\beta$ is boundary parallel, as certified by disk $E$ and $\operatorname{arc} \beta^{\prime}$, we may first assume $\operatorname{int}(E) \subset \operatorname{int}\left(H_{\Gamma} \backslash D_{\Gamma}\right)$, and then we may straighten $E$ so that, near $\left(\partial H_{\Gamma}\right) \backslash D_{\Gamma}, E$ is part of the cone over $\beta^{\prime}$. The arc $\beta$ may be slid along $E$ near to $\beta^{\prime}$, then isotoped along the cone over $\beta^{\prime}$ until it coincides with $\alpha$.

In our construction, we are mainly interested in a curve of the form $(O v \cup O w) / \epsilon_{m}$, where $O$ is the center of $B$ and $v$ and $w$ are vertices of $\Gamma$, all of which are identified by $\epsilon_{m}$ to a single vertex $x$ in $H_{\Gamma}$. The set $(O v \cup O w) \cap H_{\Delta}$ is immediately apparent. However, we must identify $\beta=\left(v^{\prime} v \cup w^{\prime} w\right) / \epsilon_{m}$, where $v^{\prime}=\left(\frac{3}{4}\right) \cdot v$ and $w^{\prime}=\left(\frac{3}{4}\right) \cdot w$. The images of $v$ and $w$ in $H_{\Gamma}$ are the single vertex $x$ of $H_{\Gamma}$, and the image of $\beta$ is a cone from $x$ in the cone structure on $H_{\Gamma} \backslash D_{\Gamma}$. Therefore, by Lemma 3.6, to identify $\beta$ it suffices to find a boundary parallel arc in $H_{\Gamma}$ with endpoints $v^{\prime}$ and $w^{\prime}$.

The vertices $v^{\prime}=\left(\frac{3}{4}\right) \cdot v$ and $w^{\prime}=\left(\frac{3}{4}\right) \cdot w$ lie in $\mathbb{R}^{2} \times\{0\}$, disjoint from the disks $\left(\frac{3}{4}\right) \cdot D_{\Delta}$, i.e., the attaching disks of the handles of $D_{\Delta}$ in $\mathbb{R}^{2} \times\{0\}$. Hence, there is an arc $\alpha^{\prime}$ in $\left(\mathbb{R}^{2} \times\{0\}\right) \backslash\left(\frac{3}{4}\right) \cdot D_{\Delta}$ from $v^{\prime}$ to $w^{\prime}$. Take the product of $\alpha^{\prime}$ and a small closed interval with left endpoint 0 in $\mathbb{R}^{2} \times[0, \infty) \subset \mathbb{R}^{3}$. We obtain a disk $D$ in the handlebody $H$ that is the closure of $\mathbb{S}^{3} \backslash H_{\Delta}$. This disk exhibits the complementary arc $\alpha \subset \partial D$ as boundary parallel in $H$. We fix this arc and construct the handlebodies $H^{\prime}$ and $H^{\prime \prime}$. Provided that the annuli and tori used in constructing $H^{\prime}$ from $H$ are chosen close enough to the curves $\delta=\partial D_{\Delta}$ to avoid $D$, the disk $D$ will also certify that $\alpha$ misses the handle disks $E_{i}$ of $H^{\prime}$ so that $\alpha$ is boundary parallel in $H^{\prime}$. If the annuli $A_{j}$ and tori $U_{j}$ are chosen close enough to $\gamma=\partial D_{\Gamma}$ to avoid $\alpha$ (but not $D$ ), then the homeomorphism $\Phi: H^{\prime} \rightarrow H^{\prime \prime}$ will fix $\alpha$ and will take the disks $E_{i}$ to handle disks for $H^{\prime \prime}$, and the disk $\Phi(D)$ will show that $\alpha$ is boundary parallel in $H^{\prime \prime}$. Thus $\left(O v^{\prime} \cup O w^{\prime}\right) \cup \alpha$ represents the curve $(O v \cup O w) / \epsilon_{m}$ as desired.

Now we add this axis to our surgery descriptions. For the simplest construction, with equator $e$ and longitudinal arc $N S$, and handle curves as shown in Figure 8, the surgery description is obtained by pushing $\delta_{0}$ slightly into $H$. Let $N^{\prime}=\left(\frac{3}{4}\right) \cdot N$ and $S^{\prime}=\left(\frac{3}{4}\right) \cdot S$ on $\left(\frac{3}{4}\right) \cdot \Gamma \subset \mathbb{R}^{2} \times\{0\}$. The $\operatorname{arc}\left(O N^{\prime} \cup O S^{\prime}\right)$ runs below the plane $\mathbb{R}^{2} \times\{0\}$ in $H_{\Delta}$. To find the arc $\alpha$, we take an arc $\alpha^{\prime}$ from $N^{\prime}$ to $S^{\prime}$ in $\mathbb{R}^{2} \times\{0\}$ disjoint from the handle, and, fixing the endpoints, push this above $\mathbb{R}^{2} \times\{0\}$ slightly. By the above discussion, this gives the desired arc of the axis $N S$. The surgery diagram and the axis are shown for this example in Figure 10. 


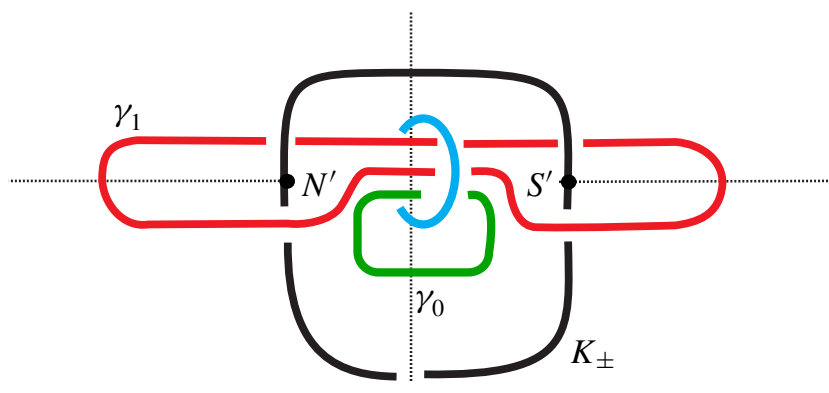

Figure 10. The surgery diagram for $K_{ \pm}, S_{1}$, and $F_{1}$.

\section{Two-bridge knots}

In this section, we prove that the image of the $N S$ axis in Figure 10 represents the figure-eight knot in $\mathbb{S}^{3}$ when the surgery coefficient is taken to be +1 , and the trefoil knot in $\mathbb{S}^{3}$ when the coefficient is taken to be -1 .

More generally, we prove that the $N S$ axis in the general construction represents a two-bridge knot in $\mathbb{S}^{3}$.

4A. Identifying the trefoil and figure-eight. We will modify the surgery diagram of Figure 10 by means of Rolfsen twists. We remind the reader of the effect of a Rolfsen twist. We assume we are given an unknotted curve $J$ with surgery coefficient $p / q$ through which pass a number of curves, some of which are surgery curves $K_{i}$ with surgery coefficients $r_{i}$, and some of which may be of interest for some other reasons, such as our knot axis. We perform an $n$-twist on $J$. The curves passing through $J$ acquire $n$ full twists as a group. The curve $J$ acquires the new surgery coefficient $p /(q+n p)$; in particular, if $p=1$, then a twist of $-q$ will change the coefficient to $\infty$, and any curve with a surgery coefficient $\infty$ can be removed from the diagram. Finally, each surgery curve $K_{i}$ that passes through $J$ acquires the new surgery coefficient $r_{i}+n \cdot \operatorname{lk}\left(J, K_{i}\right)^{2}$.

Theorem 4.1. The surgery description of $M(\epsilon, m)$ for the simple face-pairing of Figure 3 (left) yields the manifold $\mathbb{S}^{3}$. The image of the north-south axis is the trefoil knot when $m=(-1,1)$ and the figure-eight knot when $m=(1,1)$.

Proof. We apply Rolfsen twists to our surgery curves in the order $\gamma_{1}, \gamma_{0}$, and $\delta^{\prime}$ to change their surgery coefficients, one after the other, to $\infty$. We trace the effect on the axis $K_{ \pm 1}$, and show this in Figure 11 .

In detail, we first perform a -1 Rolfsen twist on $\gamma_{1}$. This changes the surgery coefficient on $\gamma_{1}$ to $\infty$ so that $\gamma_{1}$ can be removed from the diagram. In the process, one negative full twist is added to the axis representing $K_{ \pm}$. 


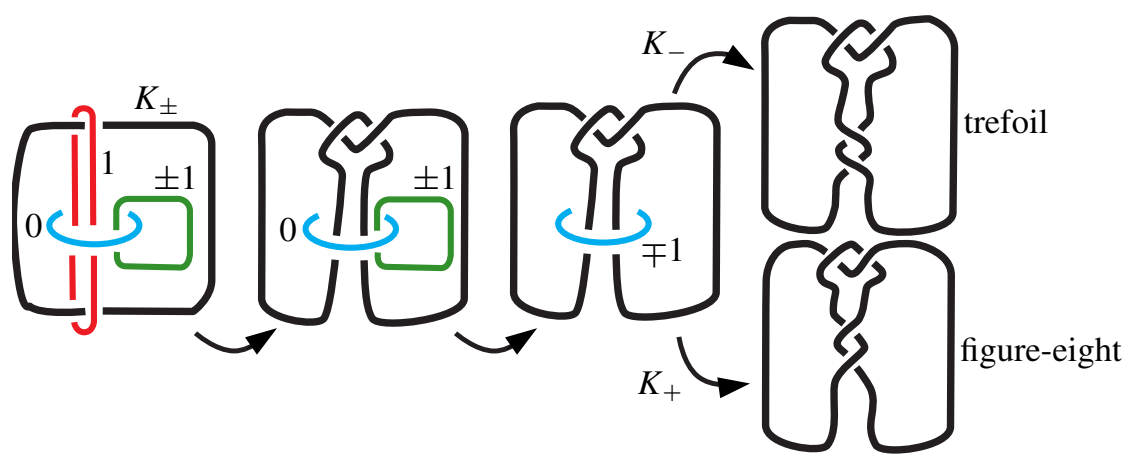

Figure 11. Analyzing $S_{1}, F_{1}, K_{-}$, and $K_{+}$.

We next perform a Rolfsen twist on $\gamma_{0}$ to change its surgery coefficient to $\infty$ so that it too can be removed from the diagram. If the coefficient on $\gamma_{0}$ was originally 1 , this twist must be $\mathrm{a}-1$ twist. If the coefficient on $\gamma_{0}$ was originally -1 , this twist must be $\mathrm{a}+1$ twist. The coefficient of this twist is added to the 0 coefficient on the $\delta^{\prime}$ curve. The axis is not affected.

Finally, we perform a Rolfsen twist on $\delta^{\prime}$, opposite to its surgery coefficient $\mp 1$ so that its coefficient is changed to $\infty$. That makes it possible to remove $\delta^{\prime}$ from the diagram. Since the diagram is now empty, we can conclude that the quotient manifold is $\mathbb{S}^{3}$.

This last twist adds a \pm 1 full twist to the axis and results in either the trefoil knot for the $(-1,1)$ multiplier pair or the figure-eight knot for the $(1,1)$ multiplier pair.

4B. The general case. Having analyzed the simplest model face-pairing, we proceed to the general case. Thus we consider the 2-sphere $\mathbb{S}^{2}=\partial \mathbb{B}^{3}$ subdivided by one longitude, the equator $e, k \geq 0$ latitudinal circles in the northern hemisphere, and their reflections in the southern hemisphere. As usual, we pair faces by reflection in the equator. There are $k+1$ face pairs in this model face-pairing.

The general surgery description is given in Lemma 3.5, and illustrated in Figure 9. Section 3D tells us how to recognize the image of the north-south axis in this diagram. It is the union of a boundary parallel arc below the plane $\mathbb{R}^{2} \times\{0\}$ from $N^{\prime}$ to $S^{\prime}$ and a boundary parallel arc above the plane $\mathbb{R}^{2} \times\{0\}$ from $N^{\prime}$ to $S^{\prime}$. Straightening this axis curve and the surgery diagram, we obtain the diagram in Figure 12.

Recall that the integers $m_{2 i+1}$ are arbitrary - positive, negative, or zero. The integers $m_{2 i}$ are either +1 or -1 . Note that the surgery curves fall naturally into three families, each with $k+1$ curves: the $\delta$ curves, circling the handles with surgery coefficients 0 , the latitudinal curves, linking the 0 -curves together in a chain and having coefficients $1 / m_{2 i}= \pm 1$, and the longitudinal curves with coefficients 


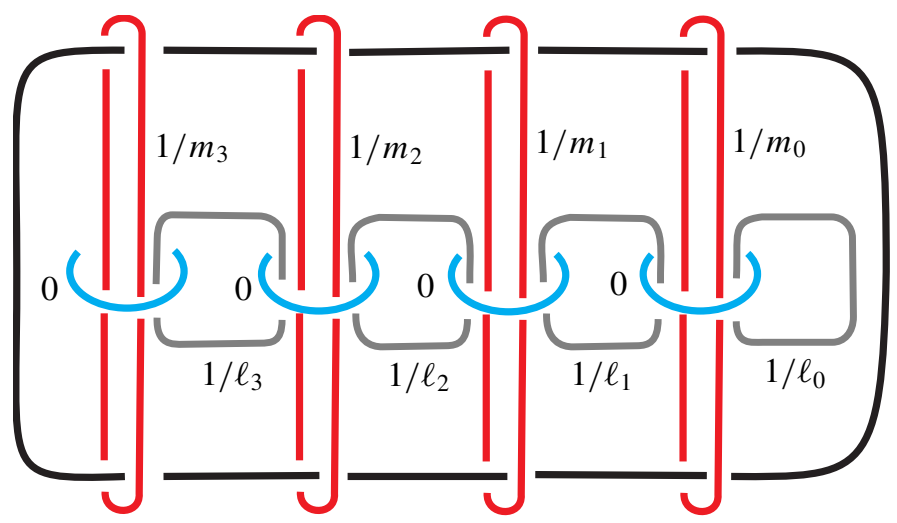

Figure 12. The surgery diagram.

$1 / m_{2 i+1}$. Each of these curve families has a natural left-to-right order, as in the figure. To simplify notation, we denote the latitudinal curves from left to right by $L_{k}, L_{k-1}, \ldots, L_{1}, L_{0}$, and let the corresponding surgery coefficients be denoted $1 / \ell_{k}, 1 / \ell_{k-1}, \ldots, 1 / \ell_{i}, 1 / \ell_{0}$, respectively (so $\ell_{i}$ now replaces notation $m_{2 i}$ ). We denote the longitudinal curves from left to right by $M_{k}, M_{k-1}, \ldots, M_{1}, M_{0}$, and renumber their surgery coefficients to be $1 / m_{k}, 1 / m_{k-1}, \ldots, 1 / m_{1}, 1 / m_{0}$. We denote the $\delta$ curves from left to right by $O_{k}, O_{k-1}, \ldots, O_{1}, O_{0}$, with surgery coefficients 0 . This decreasing order of subscripts is suggested by the usual inductive description of a rational tangle and the associated continued fraction $\left[a_{0}, a_{1}, \ldots, a_{n}\right]=a_{0}+1 /\left(a_{1}+1 /\left(a_{2}+\cdots+1 / a_{n}\right)\right)$, where the coefficient $a_{n}$ represents the first twist made in the construction and $a_{0}$ represents the last twist.

We now prove the following theorem.

Theorem 4.2. The bitwist manifold $M(\epsilon, m)$ is the 3-sphere $\mathbb{S}^{3}$. The image of the north-south axis in $\mathbb{S}^{3}$ is the two-bridge knot which is the numerator closure of the tangle $T(a / b)$ whose rational number invariant $a / b$ is

$$
\left[2 \ell_{0}, 2 m_{0}, 2 \ell_{1}, 2 m_{1}, \ldots, 2 \ell_{k}, 2 m_{k}\right]
$$

or in continued fraction form,

$$
2 \cdot \ell_{0}+\frac{1}{2 \cdot m_{0}+\frac{1}{2 \cdot \ell_{1}+\frac{1}{2 \cdot m_{1}+\frac{1}{\ddots}}}} .
$$

Here $\ell_{0}, \ell_{1}, \ell_{2}, \ldots$ and $m_{0}, m_{1}, m_{2}, \ldots$ are the multipliers of the latitudinal and longitudinal edge cycles, respectively. 


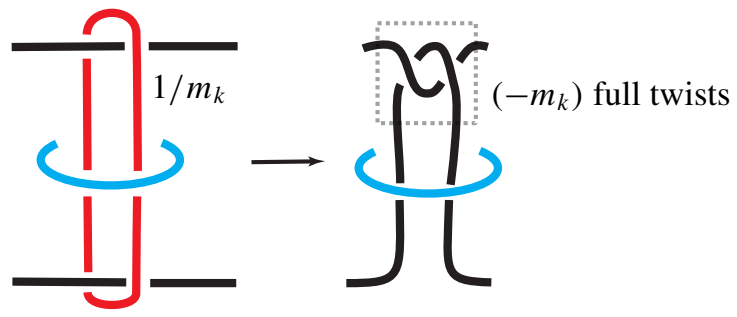

Figure 13. Removing the curve $M_{k}$ adds $-m_{k}$ horizontal twists.

Proof. We shall reduce the surgery diagram to the empty diagram by a sequence of Rolfsen twists. This will show that the quotient manifold is $\mathbb{S}^{3}$. We shall track the development of the axis as we perform those twists and show that, at each stage, the knot is a two-bridge knot. We perform the Rolfsen twists on curves in decreasing order of subscripts in the following order: $M_{k}, L_{k}, O_{k}, M_{k-1}, L_{k-1}, O_{k-1}$, etc., in order to change surgery coefficients one after the other to $\infty$. Once a coefficient is $\infty$, that curve can be removed from the diagram.

There are two cases.

Case 1: If $m_{k}=0$, so that $1 / m_{k}=\infty$, we simply remove $M_{k}$ and the axis is not affected. We may then remove $L_{k}$ and $O_{k}$ without affecting the rest of the diagram as follows. First, twist $-\ell_{k}=\mp 1$ about $L_{k}$, to give $L_{k}$ a surgery coefficient of $\infty$. This allows us to remove $L_{k}$. It also links $O_{k}$ and $O_{k-1}$ and changes the surgery coefficient on each from 0 to $-\ell_{k}$, but it does not affect the axis or the other link components. Now twist $\ell_{k}$ times about $O_{k}$. This allows us to remove $O_{k}$, returns the surgery coefficient of $O_{k-1}$ to 0 , and leaves the rest of the diagram unchanged. The diagram is now as in Figure 12, only with fewer link components. Thus we repeat the argument with this new link component. By induction, either all $m_{j}=0$, all link components can be removed, resulting in $\mathbb{S}^{3}$ with the unknot as the image of the axis, or eventually we are in case 2.

Case 2: If $m_{k} \neq 0$, we twist $-m_{k}$ times about $M_{k}$. The coefficient of $M_{k}$ then becomes $\infty$ so that $M_{k}$ can be removed from the surgery diagram. This twists two strands of the axis together as in Figure 13, introducing $-2 \cdot m_{k}$ half twists into the axis (according to our sign convention). This twist has no effect on the other curves in the diagram.

Note that the axis has formed a rational tangle at the top left of the diagram. To identify the tangle, we will use work of Kauffman and Lambropoulou [2002], with attention to orientation. Our twisting orientation agrees with theirs for horizontal twists, and so at this point, the rational tangle has continued fraction with the single entry $\left[-2 m_{k}\right]$.

The proof now proceeds by induction. We will assume that at the $j$-th step, we have a surgery diagram with image of the axis with the following properties. 


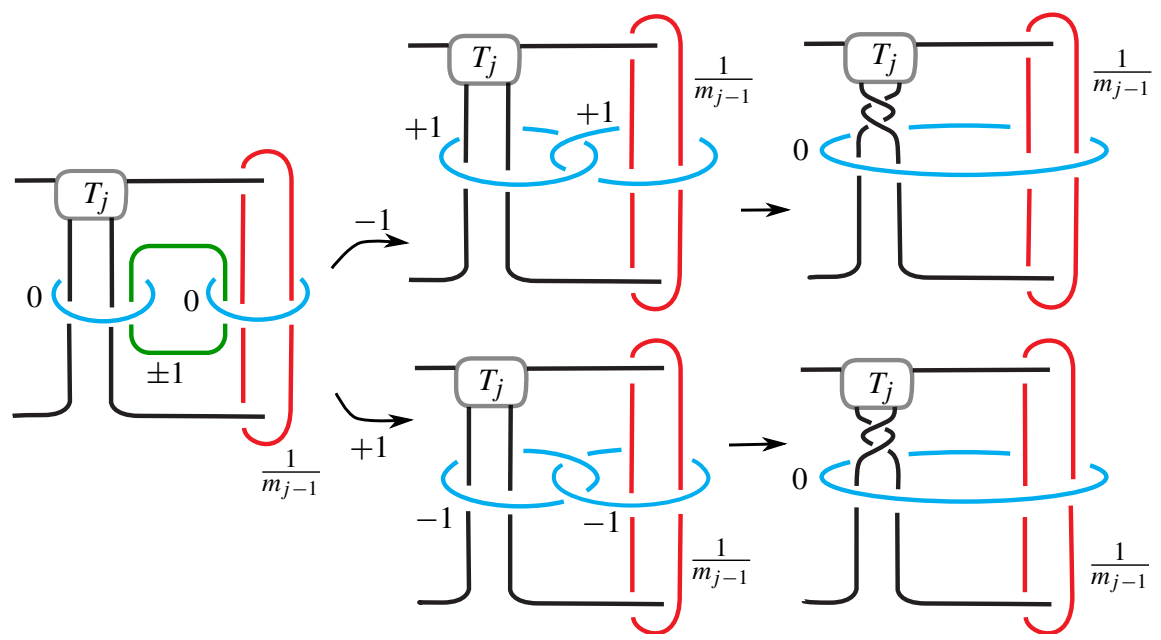

Figure 14. The effect of Rolfsen twists to remove first $L_{j}$ and then $O_{j}$.

(1) In the top-left corner, there is a rational tangle $T_{j}$ with continued fraction

$$
\left[-2 m_{j},-2 \ell_{j}, \ldots,-2 \ell_{k},-2 m_{k}\right] \text {. }
$$

(2) Two strands run from the tangle through the link component $O_{j}$.

(3) Link components $M_{k}, L_{k}, \ldots$ through $M_{j}$ have been removed.

(4) To the right, the surgery diagram is identical to the original surgery diagram, beginning with link components $L_{j}$ and running to the right through the components $M_{0}$ and $L_{0}$. That is, the link components are identical for this portion of the diagram, and the surgery coefficients are also identical.

The next step is to remove link components $L_{j}$ and $O_{j}$. This is shown in Figure 14, for both cases $\ell_{j}= \pm 1$. Carefully, we twist $-\ell_{j}$ times about $L_{j}$. The coefficient of $L_{j}$ then becomes $\infty$ so that $L_{j}$ can be removed from the surgery diagram. That twist adds $-\ell_{j}$ to the 0 surgery coefficients of $O_{j}$ and $O_{j-1}$ and links those two curves together with overcrossing having sign equal to $-\ell_{j}$. This twist has no effect on the axis. Now twist $\ell_{j}$ times about $O_{j}$. The coefficient of $O_{j}$ then becomes $\infty$ so that $O_{j}$ can be removed from the surgery diagram. The twist returns the surgery coefficient of $O_{j-1}$ back to 0 . The twist also adds $2 \cdot \ell_{j}$ half twists to the two strands of the axis that were running through $O_{j}$. Note this yields a new rational tangle, with a vertical twist added to the tangle $T_{j}$. Our twisting orientation for vertical twists is opposite that of Kauffman and Lambropoulou [2002], and so the continued fraction of this new tangle becomes $T=\left[-2 \ell_{j},-2 m_{j}, \ldots,-2 \ell_{k},-2 m_{k}\right]$.

We now need to consider $M_{j-1}$. If $m_{j-1}=0$, so its surgery coefficient is $\infty$, we simply remove $M_{j-1}$ from the surgery diagram, and we have completed the 


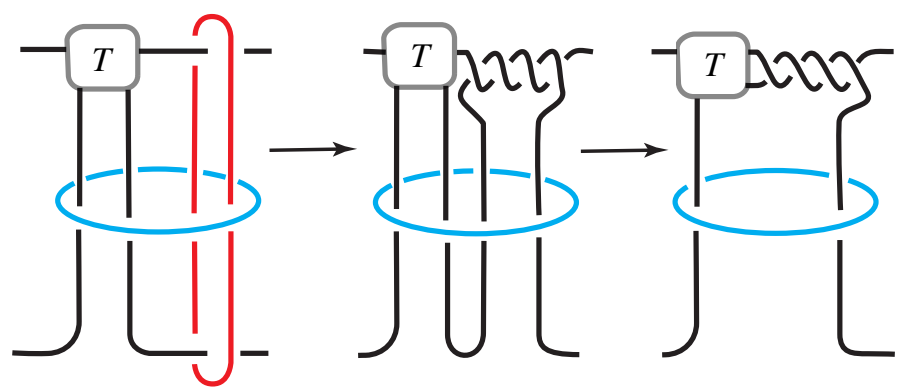

Figure 15. Removing $M_{j-1}$ through twisting.

inductive step. Otherwise, we twist $-m_{i}$ times about $M_{i}$, as in Figure 15, after which four strands of the axis pass through $O_{j-1}$. However, the central two strands can be isotoped upward through $O_{j-1}$. This adds $-2 m_{j-1}$ horizontal crossings to the tangle $T$, yielding a tangle $T_{j-1}$, and completes the inductive step.

After the final step $j=0$, we have removed all $M_{j}, L_{j}, O_{j}$ from the surgery diagram, yielding $\mathbb{S}^{3}$, and our axis has become the denominator closure of a rational tangle $T(c / d)$ with continued fraction

$$
\left[-2 \ell_{0},-2 m_{0}, \ldots,-2 \ell_{k},-2 m_{k}\right]=\frac{1}{-2 \ell_{0}+\frac{1}{-2 m_{0}+\frac{1}{-2 \ell_{1}+\frac{1}{-2 m_{1}+\frac{1}{\ddots}}}}} .
$$

The continued fraction begins with $1 /\left(-2 \ell_{0}+\cdots\right)$ instead of $-2 \cdot \ell_{0}+\cdots$ because $\ell_{0}$ corresponds to a vertical twist. Loosely speaking, horizontal twists correspond to addition and vertical twists correspond to addition and inversion. Hence our knot is the numerator closure of the tangle $T(a / b)$ with $a / b=-d / c$, as in the statement of the theorem.

Recall from Section 1 that a multiplier function $m$ with values $m_{0}, \ldots, m_{2 k+1}$ is normalized if $m_{2 k+1} \neq 0$, and if $m_{2 i+1}=0$ for some $i \in\{0, \ldots, k-1\}$, then $m_{2 i}=m_{2 i+2}$. The following example helps to motivate this definition.

Example 4.3. Figure 16 shows an example arising from multipliers

$$
\begin{aligned}
& m_{6}=3, \quad m_{5}=0, \quad m_{4}=0, \quad m_{3}=2, \quad m_{2}=-3, \quad m_{1}=0, \quad m_{0}=2, \\
& \ell_{6}=1, \quad \ell_{5}=-1, \quad \ell_{4}=-1, \quad \ell_{3}=-1, \quad \ell_{2}=1, \quad \ell_{1}=1, \quad \ell_{0}=1 .
\end{aligned}
$$

In the notation of the previous paragraph, the multiplier function has values $\ell_{0}, m_{0}, \ell_{1}, m_{1}, \ldots, m_{6}$. This multiplier function is not normalized since $\ell_{5}=-\ell_{6}$ even though $m_{5}=0$. As a result, the second vertical twist cancels the first one, and so 


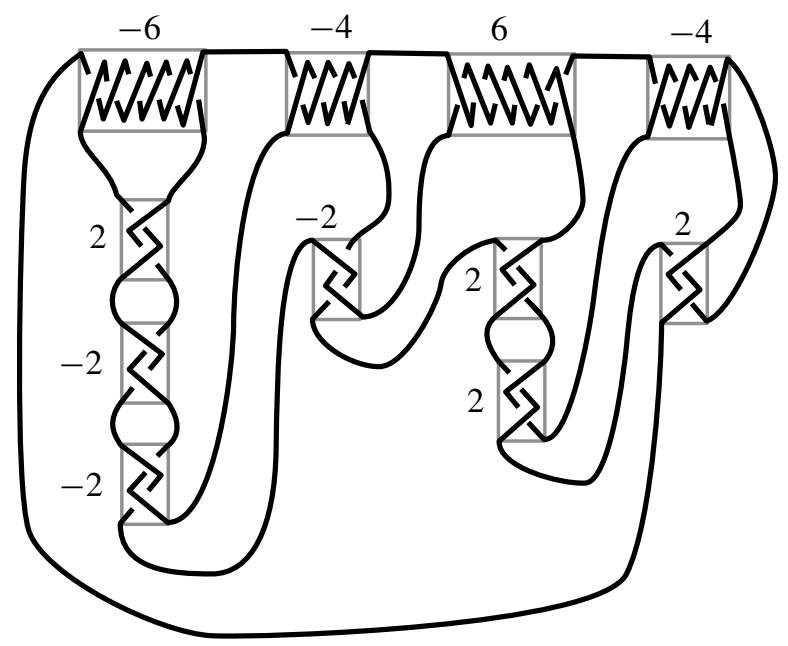

Figure 16. An example.

they can be eliminated. This is consistent with the fact that $x+1 /(0+1 / y)=x+y$, so that a continued fraction with a term equal to 0 can be simplified. Also notice that if $m_{6}=0$ instead of $m_{6}=3$, then the first three vertical twists can be untwisted, and so they can be eliminated. This is consistent with the fact that $x+1 /\left(y+\frac{1}{0}\right)=x$.

Corollary 4.4. Every normalized multiplier function yields a nontrivial two-bridge knot. Every nontrivial two-bridge knot $K$ is realized by either one or two normalized multiplier functions. Furthermore, if $K$ is the numerator closure of the tangle $T(a / b)$, then it has exactly one such realization if and only if $b^{2} \equiv 1 \bmod a$.

Proof. Note that our construction allows us to obtain any two-bridge knot with a rational invariant made only of even integers, by choosing $m_{j}=0$ appropriately. On the other hand, it is a classical result that any rational number $p / q$ with $p$ odd and $q$ even has a continued fraction expansion of the form $\left[2 a_{0}, \ldots, 2 a_{n}\right]$ with $n$ odd. This result can also be derived by a modification of the Euclidean algorithm. The corollary then follows from Theorem 4.2 and standard results involving two-bridge knots, many of which are contained in [Bleiler and Moriah 1988] and [Kauffman and Lambropoulou 2002].

\section{Cyclic presentations}

Let $M_{n}\left(K_{m}\right)$ denote the $n$-fold branched cyclic covering of $\mathbb{S}^{3}$, branched over the two-bridge knot $K_{m}$ realized by the multiplier $m$. It is known (see [Cavicchioli et al. 1999a]) that the fundamental group $G_{n}$ of $M_{n}\left(K_{m}\right)$ has a cyclic presentation. We shall show here that the bitwist representation of $M_{n}\left(K_{m}\right)$ easily leads to the same result. 
Definition 5.1. Let $X=\left\{x_{1}, \ldots, x_{n}\right\}$ be a finite alphabet. Let $\phi$ denote the cyclic permutation of $X$ that takes each $x_{i}$ to $x_{i+1}$, with subscripts taken modulo $n$. Let $W(X)$ denote a finite word in the letters of $X$ and their inverses. Then the group presentation

$$
\left\langle X \mid W(X), \phi(W(X)), \ldots, \phi^{n-1}(W(X))\right\rangle
$$

is called a cyclic presentation.

Theorem 5.2. The fundamental group of the $n$-th branched cyclic covering of $\mathbb{S}^{3}$, branched over a two-bridge knot $K$, has a cyclic presentation.

Equivalently, the group $G_{n}=\pi_{1}\left(M_{n}\left(K_{m}\right)\right)$ has a cyclic presentation. Before giving the proof, we recall the algorithm that gives a presentation for the fundamental group of the bitwist manifold $M(\epsilon, m)$. We work with the model faceted 3-ball. We assign a generator $x(f)$ to each face $f$. We will need to assign a word $W(f, e)$ to each pair $(f, e)$ consisting of a face $f$ and boundary edge $e$ of $f$, and a word $W(f)$ to each face $f$.

If $f$ is a face, denote the matching face by $f^{-1}$. Then $x\left(f^{-1}\right)=x(f)^{-1}$. If $f$ is a face and $e$ is a boundary edge of $f$, then there is a (shortest) finite sequence $(f, e)=\left(f_{1}, e_{1}\right),\left(f_{2}, e_{2}\right), \ldots,\left(f_{k}, e_{k}\right)=(f, e)$ such that $\epsilon\left(f_{i}\right)$ takes $e_{i}$ onto $e_{i+1}$ and takes $f_{i}$ onto the face across $e_{i+1}$ from $f_{i+1}$. We define $W(f, e)$ to be the word $x\left(f_{1}\right) \cdot x\left(f_{2}\right) \cdots x\left(f_{k-1}\right)$. Finally, if $f$ is a face and $e_{1}, e_{2}, \ldots, e_{j}$ are the edges of $f$, in order, with assigned multipliers $m_{1}, m_{2}, \ldots, m_{j}$, then we assign $f$ the word

$$
W(f)=W\left(f, e_{1}\right)^{m_{1}} \cdot W\left(f, e_{2}\right)^{m_{2}} \cdots W\left(f, e_{j}\right)^{m_{j}} .
$$

The next lemma follows from standard results. See also [Cannon et al. 2002, Theorem 4.8].

Lemma 5.3. The group $\pi_{1}(M(\epsilon, m))$ has presentation

$$
\langle x(f), f \text { a face }| W(f), f \text { a face }\rangle
$$

Proof of Theorem 5.2. We begin with a model faceted 3-ball and multipliers $\ell_{0}, m_{0}, \ldots, \ell_{k}, m_{k}$ used to construct $M_{1}\left(K_{m}\right)$ in Section 4B. We take its $n$-fold branched cyclic cover branched over the north-south axis. We label the faces of the northern hemisphere $x(i, j)$ as in Figure 17.

We use the same labels $x(i, j)$ as group generators. The corresponding faces and generators for the southern hemisphere are $x(i, j)^{-1}$. We distinguish three types of faces: those bordering on the equator, which are designated as type 0 , those touching the poles, which are designated as type 2 , and all others, designated type 1 . We initially assume that $k>0$ so that we don't have faces that are both type 0 and type 2 . Since edge classes have size 1 or size 2 , the words associated with a face-edge pair have length 1 or length 2 . Figure 18 shows edges of the three types 


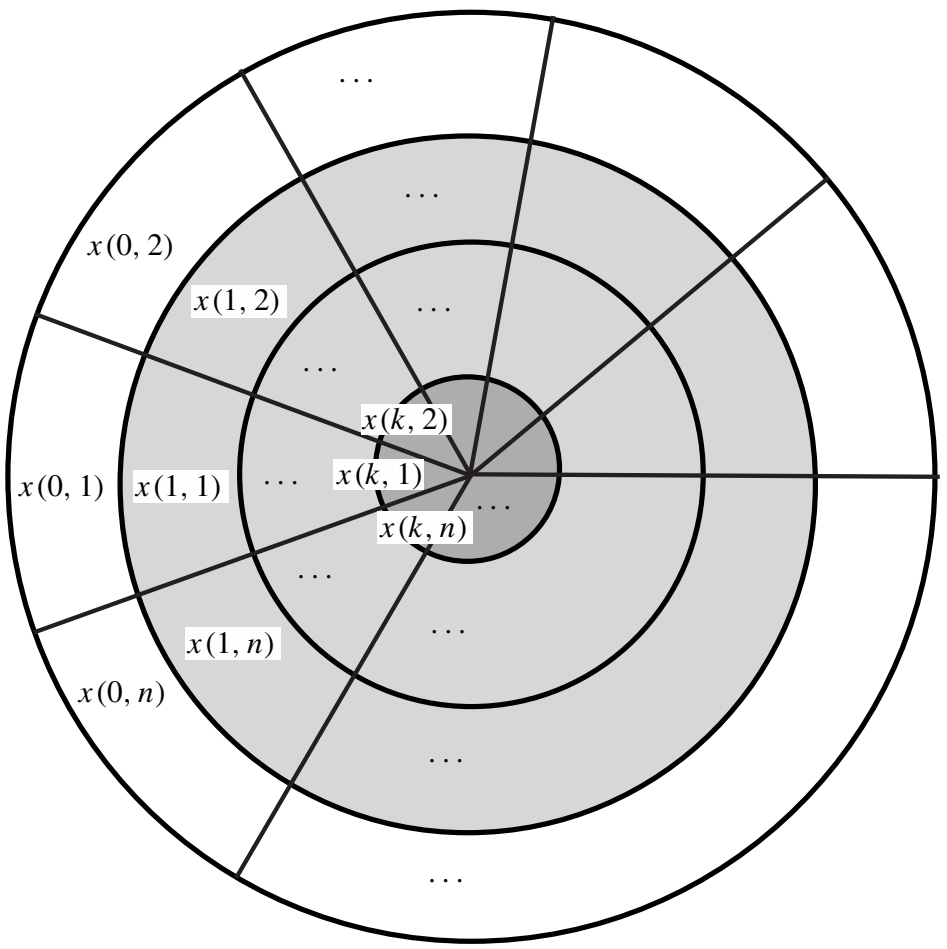

Figure 17. The model for the $n$-fold branched cyclic cover, with the face generators labeled $x(i, j)$. Faces of type 0 are shaded white, faces of type 1 are shaded light gray, and faces of type 2 are shaded darker gray.
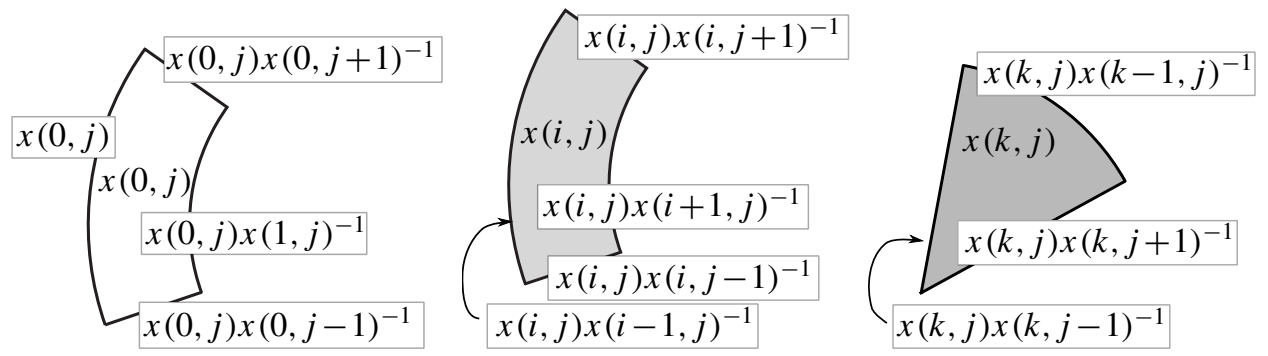

Figure 18. A face of type 0, with face-edge words (left), a face of type 1 (middle), and a face of type 2 (right).

of faces labeled with those face-edge words. These words are then raised to the appropriate powers and multiplied together to give the word associated with the corresponding face. We call these words $R(i, j)$ 's since they are the relators of the 
fundamental group. We have

$$
\begin{aligned}
& R(0, j)=[x(0, j)]^{\ell_{0}}\left[x(0, j) x(0, j+1)^{-1}\right]^{m_{0}} \\
& \times\left[x(0, j) x(1, j)^{-1}\right]^{\ell_{1}}\left[x(0, j) x(0, j-1)^{-1}\right]^{m_{0}}, \\
& R(i, j)=\left[x(i, j) x(i-1, j)^{-1}\right]^{\ell_{i}} {\left[x(i, j) x(i, j+1)^{-1}\right]^{m_{i}} } \\
& \times {\left[x(i, j) x(i+1, j)^{-1}\right]^{\ell_{i+1}}\left[x(i, j) x(i, j-1)^{-1}\right]^{m_{i}}, } \\
& R(k, j)=\left[x(k, j) x(k-1, j)^{-1}\right]^{\ell_{k}}\left[x(k, j) x(k, j+1)^{-1}\right]^{m_{k}}\left[x(k, j) x(k, j-1)^{-1}\right]^{m_{k}} .
\end{aligned}
$$

We conclude that the fundamental group has a presentation

$$
\langle x(i, j) \mid R(i, j), i=0, \ldots, k, j=1, \ldots, n\rangle .
$$

Since each of the multipliers $\ell_{0}, \ell_{1}, \ldots, \ell_{k}$ is either +1 or -1 , the letter $x(1, j)^{ \pm 1}$ appears at most once in the relator $R(0, j)$. Similarly, the letter $x(i, j)^{ \pm 1}$ appears at most once in the relator $R(i-1, j)$, for $i=2, \ldots, k-1$, and the letter $x(k, j)^{ \pm 1}$ appears at most once in the relator $R(k-1, j)$. Hence, these relators may be solved for $x(1, j), x(2, j), \ldots, x(k, j)$ iteratively, and then these relators and generators may be removed. The only generators remaining are the generators $x(0, j)$, with $j=1, \ldots, n$; and, with appropriate generator substitutions made, the only remaining relators are the relators $R(k, j)$. The presentation

$$
\langle x(0, j) \mid R(k, j), j=1, \ldots, n\rangle
$$

is clearly a cyclic presentation.

Finally, if $k=0$, then every face is both type 0 and type 2 . In this case the presentation is $\langle x(0, j) \mid R(0, j), j=1, \ldots, n\rangle$, which is cyclic.

5A. The Fibonacci and Sieradski manifolds. Recall from Section 1 that the $n$-th branched cyclic cover $S_{n}$ of the trefoil knot is called the $n$-th Sieradski manifold. The $n$-th branched cyclic cover $F_{n}$ of the figure-eight knot is called the $n$-th Fibonacci manifold.

We illustrate the above group calculations by proving a well-known theorem.

Theorem 5.4. The fundamental group $\pi_{1}\left(F_{n}\right)$ is the $2 n$-th Fibonacci group with presentation

$$
\left\langle x_{1}, \ldots, x_{2 n} \mid x_{1} x_{2}=x_{3}, x_{2} x_{3}=x_{4}, \ldots, x_{2 n-1} x_{2 n}=x_{1}, x_{2 n} x_{1}=x_{2}\right\rangle .
$$

The fundamental group $\pi_{1}\left(S_{n}\right)$ is the $n$-th Sieradski group with presentation

$$
\left\langle y_{1}, \ldots, y_{n} \mid y_{1}=y_{2} y_{n}, y_{2}=y_{3} y_{1}, y_{3}=y_{4} y_{2}, \ldots, y_{n}=y_{1} y_{n-1}\right\rangle \text {. }
$$

Proof. The faceted 3-ball that serves as the model for the face-pairings is the same for both manifolds; it is as in Figure 17 with $k=0$, so without interior latitudinal circles. 
For the Fibonacci manifolds, we label the faces of the northern hemisphere as $x(2), x(4), \ldots, x(2 n)$. All subscript calculations are modulo $2 n$. We obtain the following cyclic presentation for the fundamental group:

$$
\left\langle x(2), x(4), \ldots, x(2 n) \mid x(2 j) \cdot\left[x(2 j) x(2 j+2)^{-1}\right] \cdot\left[x(2 j) x(2 j-2)^{-1}\right]\right\rangle,
$$

with $j=1,2, \ldots, n$. We can then introduce intermediate generators $x(2 j-1)=$ $x(2 j-2)^{-1} \cdot x(2 j)$. The presentation becomes the standard presentation for the $2 n$-th Fibonacci group, as desired:

$$
\langle x(1), \ldots, x(2 n) \mid x(i+2)=x(i) \cdot x(i+1)\rangle .
$$

For the Sieradski manifolds, we label the faces of the northern hemisphere as $y(1), y(2), \ldots, y(n)$. Subscript calculations are modulo $n$. We obtain the following cyclic presentation for the fundamental group:

$$
\left\langle y(1), \ldots, y(n) \mid y(j)^{-1} \cdot\left[y(j) y(j+1)^{-1}\right] \cdot\left[y(j) y(j-1)^{-1}\right], j=1, \ldots, n\right\rangle,
$$

or, reversing the order of the subscripts so that $x(1)=y(n), \ldots, x(n)=y(1)$,

$$
\langle x(1), \ldots, x(n) \mid x(i)=x(i-1) \cdot x(i+1)\rangle,
$$

the standard presentation for the $n$-th Sieradski group.

5B. Branched cyclic covers with periodic homology. In this section we consider first homology groups of our cyclic branched covers of $\mathbb{S}^{3}$. This is a topic which has received and still receives considerable attention. There are two very different behaviors. The first homology groups of the $n$-fold cyclic covers $M_{n}$ of $\mathbb{S}^{3}$ branched over a knot $K$ are either periodic in $n$ or their orders grow exponentially fast. Specifically, Gordon [1972] proved that when the roots of the Alexander polynomial of $K$ are all roots of unity, then $\mathrm{H}_{1}\left(M_{n}, \mathbb{Z}\right)$ is periodic in $n$. Riley [1990] and, independently, González-Acuña and Short [1991] proved that if the roots of the Alexander polynomial are not all roots of unity, then the finite values of $\mathrm{H}_{1}\left(M_{n}, \mathbb{Z}\right)$ grow exponentially fast in $n$. Silver and Williams [2002] extended these results to links and replaced "finite values" with "orders of torsion subgroups". See also [Le 2009; Bergeron and Venkatesh 2013; Brock and Dunfield 2015] for more recent results and conjectures on this topic.

We are particularly fascinated by the first homology of the branched cyclic covers of $\mathbb{S}^{3}$ branched over the knots that are two-strand braids. These knots are the only two-bridge knots that are not hyperbolic.

The northern hemisphere of the model before bitwisting looks like Figure 17. We construct the $n$-fold branched cyclic cover of $\mathbb{S}^{3}$, branched over a knot that is a two-strand braid, by using $k \geq 0$ latitudes and $n$ longitudes in the open northern hemisphere, assigning multipliers -1 to the latitudinal edges, and assigning 
multipliers +1 to all longitudinal edges. We calculate the fundamental group as in the proof of Theorem 5.2 and transform it into a cyclic presentation as explained there. We then abelianize, and let $a_{0}, a_{1}, \ldots, a_{2 k+2}$ denote the exponent sums of the generators in the defining cyclic word $W$.

We very briefly indicate by diagram how these integers may be computed. Every relator corresponds to a diagram as follows:

$$
\begin{aligned}
& \begin{array}{cc|rrc} 
& & j-1 & j & j+1 \\
\cline { 2 - 4 } R(k, j) & k & 1 & -1 & 1 \\
& k-1 & & -1 &
\end{array} \\
& \begin{array}{cc|rrc} 
& & j-1 & j & j+1 \\
\cline { 2 - 4 } R(i, j) & i+1 & & -1 & \\
& i & 1 & 0 & 1 \\
& i-1 & & -1 &
\end{array} \\
& \begin{array}{lr|rrc} 
& & j-1 & j & j+1 \\
\cline { 2 - 5 } R(0, j) & 1 & & -1 & \\
& 0 & 1 & 0 & 1
\end{array}
\end{aligned}
$$

We begin with the diagram for $R(k, j)$ and use the diagrams for $R(k-1, j)$, $R(k-2, j), \ldots$ to successively transform the entries in rows $k, k-1, \ldots, 1$ to 0 . The defining cyclic word is the final result in row 0 .

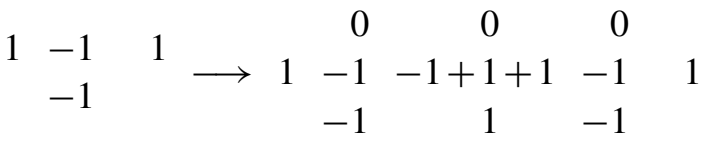

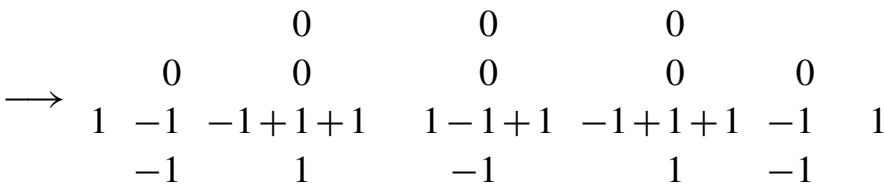

We find that the polynomial $a_{0}+a_{1} \cdot t+\cdots+a_{2 k+2} \cdot t^{2 k+2}$ is the cyclotomic polynomial

$$
1-t+t^{2}-t^{3}+\cdots-t^{2 k+1}+t^{2 k+2} \text {. }
$$

(If $2 k+3>n$, then the polynomial folds on itself because powers are to be identified modulo $n$. However, once $n \geq 2 k+3$, there is no folding.) 
Remark 5.5. The computation indicated by the diagram is a continued fraction algorithm. For the fundamental group of a general two-bridge knot, the corresponding polynomial may be taken to be the numerator of the continued fraction

$$
Q_{0}-\frac{1}{Q_{1}-\frac{1}{Q_{2}-\frac{1}{\ddots \cdot-\frac{1}{Q_{k}}}}}
$$

where

$$
Q_{i}(t)=m_{i} t-\left(\ell_{i}+\ell_{i+1}+2 m_{i}\right)+m_{i} t^{-1} \text { for } 0 \leq i \leq k-1
$$

and

$$
Q_{k}(t)=m_{k} t-\left(\ell_{k}+2 m_{k}\right)+m_{k} t^{-1} .
$$

We shall prove that, for a given knot realized as a two-strand braid, the abelianizations of the fundamental group of the $n$-fold branched cover are periodic functions of $n$. However, as a warm up, we use row reduction of the presentation matrix to prove the much easier theorem that no two of the Fibonacci groups $F(n)$ are isomorphic for $n>1$ since no two of the abelianizations have the same order. Johnson [1976, page 35] poses this problem as an exercise and suggests using the two-variable presentation of the group. We use the $n$-variable presentation and note that the Fibonacci numbers $f_{0}=0, f_{1}=1, f_{2}=1, f_{3}=2, \ldots$ appear in a very natural way. In this case we have the behavior of exponential growth of orders.

Theorem 5.6. Let

$$
\left.F(n)=\left\langle x_{1}, x_{2}, \ldots, x_{n}\right| x_{i} x_{i+1}=x_{i+2} \text { for all } i\right\rangle,
$$

with subscripts calculated modulo $n$. For odd $n$, the order of the abelianization is the sum $f_{n-1}+f_{n+1}$ of two Fibonacci numbers. For even $n$, the order is $f_{n-1}+f_{n+1}-2$.

Remark 5.7. Recall that for even $n$ these abelianizations are the first homology groups of the Fibonacci manifolds. This theorem gives successive orders of $1,1,4,5,11,16,29,45,76,121, \ldots$ for the abelianizations of the Fibonacci groups. It is clear from the definition of the Fibonacci numbers that these numbers are strictly increasing after the numbers 1,1 . These numbers are also known as the associated Mersenne numbers [Sloane and Guy 1991]. The sums $f_{n-1}+f_{n+1}$ are also known as Lucas numbers. 
Proof. The presentation matrix has the form

$$
\left(\begin{array}{rrrrrrrrr}
1 & 1 & -1 & 0 & 0 & \cdots & 0 & 0 & 0 \\
0 & 1 & 1 & -1 & 0 & \cdots & 0 & 0 & 0 \\
0 & 0 & 1 & 1 & -1 & \cdots & 0 & 0 & 0 \\
& & \cdots & & & \cdots & & \cdots & \\
0 & 0 & 0 & 0 & 0 & \cdots & 1 & 1 & -1 \\
-1 & 0 & 0 & 0 & 0 & \cdots & 0 & 1 & 1 \\
1 & -1 & 0 & 0 & 0 & \cdots & 0 & 0 & 1
\end{array}\right) .
$$

The absolute value of the determinant of this matrix is the order of the abelianization of the group unless the determinant is 0 . In that case, the group is infinite. The goal is to move the entries in the lower-left corner to the right by adding multiples of the upper rows. These operations do not change the determinant.

We use the upper rows in descending order, with each successive row moving the lower-left $2 \times 2$ matrix one column to the right. We first trace the evolution of the two entries in the next-to-last row:

$$
(-1,0) \rightarrow(1,-1) \rightarrow(-2,1) \rightarrow(3,-2) \rightarrow(-5,3) \rightarrow(8,-5) \rightarrow \cdots .
$$

The reader will easily identify the first in the $k$-th pair as $(-1)^{k} f_{k}$, and the second as $(-1)^{k-1} f_{k-1}$. Since the second of these, namely $(1,-1)$, coincides with the first pair in the bottom row, we see that the bottom row evolves just one step ahead of the next-to-last row. Thus after $k$ moves, the $2 \times 2$ matrix evolves into the matrix

$$
\left(\begin{array}{cc}
(-1)^{k} f_{k} & (-1)^{k-1} f_{k-1} \\
(-1)^{k+1} f_{k+1} & (-1)^{k} f_{k}
\end{array}\right),
$$

which has determinant $f_{k}^{2}-f_{k+1} \cdot f_{k-1}=(-1)^{k-1}$. After the appropriate number of moves, this matrix will be added to the matrix

$$
\left(\begin{array}{ll}
1 & 1 \\
0 & 1
\end{array}\right)
$$

from the lower-right corner to form the very last lower-right-corner matrix

$$
\left(\begin{array}{cc}
(-1)^{k} f_{k}+1 & (-1)^{k-1} f_{k-1}+1 \\
(-1)^{k+1} f_{k+1} & (-1)^{k} f_{k}+1
\end{array}\right) .
$$

The matrix then has determinant

$$
\begin{aligned}
{\left[f_{k}^{2}+2 \cdot(-1)^{k} \cdot f_{k}+1\right]-\left[f_{k+1} \cdot f_{k-1}+(-1)^{k+1} f_{k+1}\right] } & \\
& =(-1)^{k+1}+1+(-1)^{k}\left[f_{k}+f_{k+2}\right] .
\end{aligned}
$$

The absolute value of this determinant is the order of the abelianization, and since the last value of $k$ is $n-1$, it agrees with the value claimed in the theorem. 


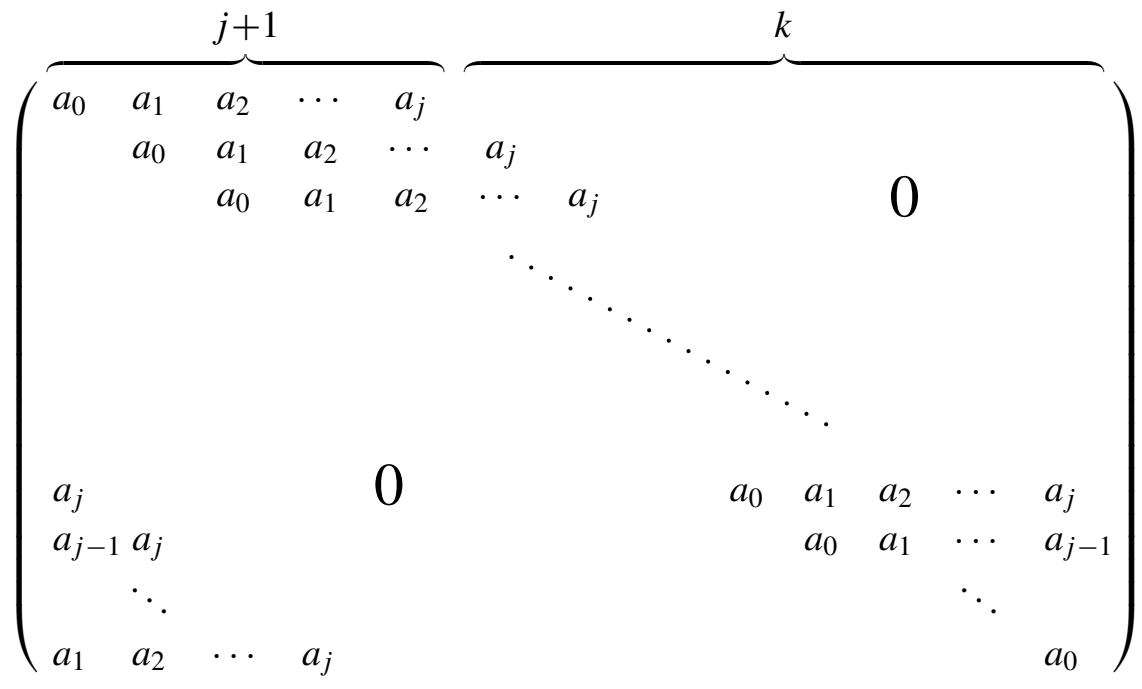

Figure 19. The relator matrix for $n=j+1+k$.

For the moment, we fix two integers $j>0$ and $k \geq 0$, and let $G_{n}$, with $n=j+1+k$, denote an abelian group with generators $x_{0}, x_{1}, x_{2}, \ldots$ such that $x_{i}=x_{i+n}$ and with relators $a_{0} \cdot x_{i}+a_{1} \cdot x_{i+1}+\cdots+a_{j} \cdot x_{i+j}$ for each $i$. Then the group has a circulant relator matrix of the form shown in Figure 19. In the following theorem we have the behavior of periodic homology groups.

Theorem 5.8. Let $j, k$, and $G_{n}$ be as immediately above, so that $n=j+1+k$. Assume that $p(t)=a_{0}+a_{1} \cdot t+\cdots+a_{j} \cdot t^{j}$ is a cyclotomic polynomial, by which we mean that there is a polynomial $q(t)=b_{0}+b_{1} \cdot t+\cdots+b_{\ell} \cdot t^{\ell}$ such that $p(t) \cdot q(t)=1-t^{j+\ell}$. Then the groups $G_{n}$ and $G_{n+j+\ell}$ are isomorphic.

Proof. We manipulate the relator matrix for $G_{n+j+\ell}$ using integral row and column operations. See Figure 19. We use the rows at the top of the matrix to remove entries from the triangle at the lower-left corner of the matrix.

Let $x$ be such an entry in row $R_{a}$. Let $R_{b}$ denote the row whose initial entry on the diagonal is above $x$. Subtract from row $R_{a}$ the expression

$$
x \cdot\left[b_{0} \cdot R_{b}+b_{1} \cdot R_{b+1}+\cdots+b_{\ell} \cdot R_{b+\ell}\right] .
$$

The effect is to move entry $x$ to the right $j+\ell$ places. Similarly, we move all entries in the lower-left triangle $j+\ell$ places to the right. Because $a_{0}= \pm 1$, we may use column operations to make every entry to the right of the first $j+\ell a_{0}$ 's equal to 0 . The lower-right $n \times n$ block of the resulting matrix is the relator matrix for $G_{n}$. The theorem follows. 
Remark 5.9. The same calculation can be carried out if the polynomial is any integer multiple $\alpha \cdot p(t)$ of a cyclotomic polynomial $p(t)$, except that the diagonal entries above the periodic box all become $\alpha$ 's. Thus the abelianization has a periodic component together with an increasing direct sum of $\mathbb{Z}_{\alpha}$ 's. It can be shown that these are the only polynomials with these periodicity properties.

Corollary 5.10. If $K$ is a knot that is a two-strand braid and $M_{n}$ is the $n$-fold cyclic branched cover of $\mathbb{S}^{3}$ over $K$, then the homology groups $H_{1}\left(M_{n}\right)$ are periodic in $n$.

Remark 5.11. Lambert [2010] explicitly calculated all of the homology groups of the branched cyclic covers of $\mathbb{S}^{3}$, branched over knots that are two-strand braids. These are the only two-bridge knots that are not hyperbolic. His tables give an explicit picture of the periodicity we have just proved. Rolfsen [1976] notes that the period for the trefoil is 6 . We shall also see that as follows.

Proof of Corollary 5.10. It suffices to find the appropriate polynomials $q(t)$, and thereby determine the period. If $p(t)=1-t+t^{2}$, as for the trefoil, then the appropriate $q(t)$ of smallest degree is $q(t)=1+t-t^{3}-t^{4}$ so that the period is $2+4=6$. With five half twists, $p(t)=1-t+t^{2}-t^{3}+t^{4}$ and $q(t)=1+t-t^{5}-t^{6}$ and the period is $4+6=10$. Each added pair of half twists in the braid adds two terms to $p(t)$, multiplies the negative entries of $q(t)$ by $t^{2}$, and increases the period by 4 .

Remark 5.12. By [Gordon 1972], the homology groups $H_{1}\left(M_{n}\right)$ of the cyclic branched covers $M_{n}$ of the complement of a knot $K$ are periodic with period dividing $m$ if and only if the first Alexander invariant (the quotient of the first two Alexander polynomials) of $K$ is a divisor of the polynomial $t^{m}-1$. Furthermore, if the first Alexander invariant is a divisor of $t^{m}-1$ and $n$ is a positive integer, then $H_{1}\left(M_{n}\right)=H_{1}\left(M_{(m, n)}\right)$, where $(m, n)$ is the greatest common divisor of $m$ and $n$. Since the first Alexander invariant of the trefoil knot is $1-t+t^{2}$, which divides $t^{6}-1$, Gordon's theorem shows that the first homology groups of the cyclic branched covers of the trefoil knot are periodic with period 6 and $H_{1}\left(S_{6 j+2}\right)=H_{1}\left(S_{6 j+4}\right)$ for all $j$.

We use the calculation of the period of the trefoil in establishing the next theorem.

Theorem 5.13. No two of the Sieradski groups are isomorphic. Hence no two of the branched cyclic covers of $\mathbb{S}^{3}$, branched over the trefoil knot, are homeomorphic.

Proof. Milnor [1975] defines the Brieskorn manifold $M(p, q, r)$ to be the orientable closed 3-manifold obtained by intersecting the complex algebraic surface given by $z_{1}^{p}+z_{2}^{q}+z_{3}^{r}=0$ with the unit sphere given by $\left|z_{1}\right|^{2}+\left|z_{2}\right|^{2}+\left|z_{3}\right|^{2}=1$. Here $p, q, r$ should be integers at least 2. Theorem 2.1 of [Cavicchioli et al. 1998], by Cavicchioli, Hegenbarth, and A. C. Kim, states that $S_{n}$ is the Brieskorn manifold $M(2,3, n)$. This follows from the fact that $S_{n}$ is the $n$-fold cyclic branched cover of $\mathbb{S}^{3}$ branched over the trefoil knot, which is the torus knot of type $(2,3)$, and 
Lemma 1.1 of [Milnor 1975], which states that the Brieskorn manifold $M(p, q, r)$ is the $r$-fold cyclic branched cover of $\mathbb{S}^{3}$ branched over a torus link of type $(p, q)$.

The first few $n$-fold cyclic covers of $\mathbb{S}^{3}$ branched over the right-hand trefoil knot are discussed in Section 10D of Rolfsen's book [1976], which begins on page 304. Here are the results.

- $n=1$ : The manifold $S_{1}$ is the 3 -sphere $\mathbb{S}^{3}$, and so $G_{1}=1$.

- $n=2$ : The manifold $S_{2}$ is the lens space $L(3,1)$, so $G_{2} \cong \mathbb{Z} / 3 \mathbb{Z}$.

- $n=3$ : The manifold $S_{3}$ is the spherical 3-manifold with fundamental group $G_{3}$ the quaternion group of order 8. It appears in Example 7.2 of [Cannon et al. 2002]. This group might be called the binary Klein 4-group.

- $n=4$ : The manifold $S_{4}$ is the spherical 3-manifold with fundamental group $G_{4}$ the binary tetrahedral group.

- $n=5$ : The manifold $S_{5}$ is the spherical 3-manifold with fundamental group $G_{5}$ the binary icosahedral group. In other words, this is the Poincaré homology sphere.

- $n=6$ : The manifold $S_{6}$ is the Heisenberg manifold. Here

$$
G_{6} \cong\langle x, y:[x,[x, y]]=[y,[x, y]]=1\rangle .
$$

Milnor [1975] proves that $M(2,3, n)$, which we know is homeomorphic to $S_{n}$, is an $\widetilde{\mathrm{SL}}(2, \mathbb{R})$-manifold for $n \geq 7$. It follows that $G_{1}, \ldots, G_{6}$ are distinct and that they are not $\widetilde{\mathrm{SL}}(2, \mathbb{R})$ manifold groups. Because of this and Milnor's result that $S_{n}$ is an $\widetilde{\mathrm{SL}}(2, \mathbb{R})$-manifold for $n \geq 7$, to prove that the groups $G_{n}$ are distinct, it suffices to prove that the groups $G_{n}$ are distinct for $n \geq 7$.

As stated on page 304 of [Rolfsen 1976], for every positive integer $n$ the first homology group $H_{1}\left(S_{n}\right)$ is $\mathbb{Z} \oplus \mathbb{Z}, 0, \mathbb{Z} / 3 \mathbb{Z}$, or $\mathbb{Z} / 2 \mathbb{Z} \oplus \mathbb{Z} / 2 \mathbb{Z}$ when $n \equiv 0, \pm 1, \pm 2$, or $3 \bmod 6$. So to prove that Sieradski groups $G_{m}$ and $G_{n}$ are distinct, we may assume that $m \equiv \pm n \bmod 6$.

For the rest of this section suppose that $n \geq 7$. Milnor [1975] (see the bottom of page 213 and Lemma 3.1) proved that $G_{n}$ is isomorphic to the commutator subgroup of the centrally extended triangle group

$$
\Gamma(2,3, n)=\left\langle\gamma_{1}, \gamma_{2}, \gamma_{3}: \gamma_{1}^{2}=\gamma_{2}^{3}=\gamma_{3}^{n}=\gamma_{1} \gamma_{2} \gamma_{3}\right\rangle
$$

Let $\Delta(2,3, n)=\left\langle\delta_{1}, \delta_{2}, \delta_{3}: \delta_{1}^{2}=\delta_{2}^{3}=\delta_{3}^{n}=\delta_{1} \delta_{2} \delta_{3}=1\right\rangle$, a homomorphic image of $\Gamma(2,3, n)$. The group $\Delta(2,3, n)$ is the group of orientation-preserving elements of the $(2,3, n)$-triangle group. Let $\Delta^{\prime}(2,3, n)$ denote the commutator subgroup of $\Delta(2,3, n)$. We see that the quotient group $\Delta(2,3, n) / \Delta^{\prime}(2,3, n)$ is isomorphic to the group generated by the elements $(1,0,0),(0,1,0)$, and $(0,0,1)$ in $\mathbb{Z}^{3}$ with 
relations corresponding to a matrix which row reduces as follows:

$$
\left[\begin{array}{lll}
1 & 1 & 1 \\
2 & 0 & 0 \\
0 & 3 & 0 \\
0 & 0 & n
\end{array}\right] \longrightarrow\left[\begin{array}{rrr}
1 & 1 & 1 \\
0 & -2 & -2 \\
0 & 3 & 0 \\
0 & 0 & n
\end{array}\right] \longrightarrow\left[\begin{array}{rrr}
1 & 1 & 1 \\
0 & -2 & -2 \\
0 & 1 & -2 \\
0 & 0 & n
\end{array}\right] \longrightarrow\left[\begin{array}{rrr}
1 & 0 & 3 \\
0 & 1 & -2 \\
0 & 0 & 6 \\
0 & 0 & n
\end{array}\right]
$$

So $\Delta(2,3, n) / \Delta^{\prime}(2,3, n)$ is a cyclic group of order $k=\operatorname{GCD}(6, n)$. This computation also shows that $\delta_{1} \in \Delta^{\prime}(2,3, n)$ if and only if $n \neq \equiv 0 \bmod 2$, that $\delta_{2} \in \Delta^{\prime}(2,3, n)$ if and only if $n \neq \equiv \bmod 3$, and that $\delta_{3}^{k}$ is the smallest power of $\delta_{3}$ in $\Delta^{\prime}(2,3, n)$. In particular $\delta_{3}^{k}$ is a nontrivial elliptic element of $\Delta^{\prime}(2,3, n)$. Every element of $\Delta^{\prime}(2,3, n)$ which commutes with $\delta_{3}^{k}$ must fix the fixed point of $\delta_{3}^{k}$. It easily follows that the center of $\Delta^{\prime}(2,3, n)$ is trivial, and in the same way that the center of $\Delta(2,3, n)$ is trivial.

Since the kernel of the homomorphism from $\Gamma(2,3, n)$ to $\Delta(2,3, n)$ is generated by the central element $\gamma_{1} \gamma_{2} \gamma_{3}$ and the center of $\Delta(2,3, n)$ is trivial, it follows that the kernel of this homomorphism is the center of $\Gamma(2,3, n)$. So $\Gamma(2,3, n)$ modulo its center is isomorphic to $\Delta(2,3, n)$. Similarly, $G_{n}$ modulo its center is isomorphic to $\Delta^{\prime}(2,3, n)$.

Now suppose that $n \equiv \pm 1 \bmod 6$. Then $G_{n}$ modulo its center is isomorphic to $\Delta^{\prime}(2,3, n)=\Delta(2,3, n)$. The largest order of a torsion element in $\Delta(2,3, n)$ is $n$. So $G_{m}$ and $G_{n}$ are distinct if $m \equiv n \equiv \pm 1 \bmod 6$. Next suppose that $n \equiv \pm 2 \bmod 6$. In this case the largest order of a torsion element in $\Delta^{\prime}(2,3, n)$ is $n / 2$. So $G_{m}$ and $G_{n}$ are distinct if $m \equiv n \equiv \pm 2 \bmod 6$. The same argument is valid if $n \equiv 3 \bmod 6$. Finally suppose that $n \equiv 0 \bmod 6$. In this case neither $\delta_{1}$ nor $\delta_{2}$ are in $\Delta^{\prime}(2,3, n)$. In this case every torsion element in $\Delta^{\prime}(2,3, n)$ is conjugate to a power of $\delta_{3}^{6}$, which has order $n / 6$. Again $G_{m}$ and $G_{n}$ are distinct if $m \equiv n \equiv 0 \bmod 6$.

\section{History}

There is a large literature concerning the Fibonacci groups, the Sieradski groups, their generalizations, cyclic presentations of groups, the relationship between cyclic presentations and branched cyclic covers of manifolds, two-bridge knots, and their generalizations. We are incapable of digesting, let alone giving an adequate summary of, this work. We plead forgiveness for having omitted important and beautiful work and for misrepresenting work that we have not adequately studied.

6A. The Fibonacci groups. John Conway told the first-named author of this paper that he created the Fibonacci group $F(5)$, with presentation

$$
\left\langle x_{1}, \ldots, x_{5} \mid x_{1} x_{2}=x_{3}, x_{2} x_{3}=x_{4}, x_{3} x_{4}=x_{5}, x_{4} x_{5}=x_{1}, x_{5} x_{1}=x_{2}\right\rangle,
$$


and asked that his graduate students calculate its structure as an exercise to demonstrate that it is not easy to read the structure of a group from a group presentation. For example, our straightforward coset enumeration program creates four layers and more than 200 vertices before the coset graph collapses to its final 11 elements. Conway [1965] presented the calculation as a problem. The definition was immediately generalized to give the group $F(n)$. Coset enumeration showed that $F(n)$ is finite for $n<6$ and for $n=7$. The Cayley graph for group $F(6)$ can be constructed systematically and recognized as a 3-dimensional infinite Euclidean group. Roger Lyndon proved, using small cancellation theory, that $F(n)$ is infinite if $n \geq 11$ (unpublished). A. M. Brunner [1974] proved that $F(8)$ and $F(10)$ are infinite. George Havas, J. S. Richardson, and Leon S. Sterling [Havas et al. 1979] showed that $F(9)$ has a quotient of order $152 \cdot 5^{18}$, and, finally, M. F. Newman [1990] proved that $F(9)$ is infinite. Derek F. Holt [1995] later reported a proof by computer that $F(9)$ is automatic, from which it could be seen directly from the word-acceptor that the generators have infinite order.

At the International Congress in Helsinki (1978), Bill Thurston was advertising the problem (eventually solved by Misha Gromov) of proving that a group of polynomial growth has a nilpotent subgroup of finite index. The first-named author brought up the example of $F(6)$ as such a group. Thurston immediately recognized the group as a branched cyclic cover of $\mathbb{S}^{3}$, branched over the figure-eight knot. And before our dinner of reindeer steaks was over, Thurston had conjectured that the even-numbered Fibonacci groups were probably also branched cyclic covers of $\mathbb{S}^{3}$, branched over the figure-eight knot. This conjecture was verified by H. M. Hilden, M. T. Lozano, and J. M. Montesinos-Amilibia [Hilden et al. 1992] and by H. Helling, A. C. Kim, and J. L. Mennicke [Helling et al. 1998]. C. Maclachlan [1995] proved that, for odd $n$, the group $F(n)$ is not a fundamental group of a hyperbolic 3-orbifold of finite volume.

6B. Sieradski manifolds. The Sieradski manifolds have a similar rich history, but not one we know as well. They were introduced by A. Sieradski [1986], who used the same faceted 3-ball that we employ, though his face-pairings were different. Richard M. Thomas [1991] showed that the Sieradski groups, which he calls $G(n)$, are infinite if and only if $n \geq 6$ and that $G(6)$ is metabelian. Cavicchioli, Hegenbarth, and A. C. Kim [Cavicchioli et al. 1998] showed that the Sieradski manifolds are branched over the trefoil knot.

6C. Cyclic presentations. Cyclic presentations are particularly interesting because of their connections with branched cyclic coverings of 3-manifolds. Fundamental results about cyclic presentations appear in the book Presentations of groups by D. L. Johnson [1976, Chapter 16]. Arye Juhász [2007] considered the question of when cyclically presented groups are finite. Andrzej Szczepański and Andrei Y. 
Vesnin [2000] asked which cyclically presented groups can be groups of hyperbolic 3-orbifolds of finite volume and which cannot. Cavicchioli and Fulvia Spaggiari [2006] showed that nonisomorphic cyclically presented groups can have the same polynomial.

6D. Dunwoody manifolds. M. J. Dunwoody [1995] managed to enumerate, with parameters, a large class of 3-manifolds admitting Heegaard splittings with cyclic symmetry. The fundamental groups were all cyclically presented. He observed that the polynomials associated with the cyclic presentations were Alexander polynomials of knots and asked whether the spaces were in fact branched cyclic covers of $\mathbb{S}^{3}$, branched over knots or links. Cavicchioli, Hegenbarth, and A. C. Kim [Cavicchioli et al. 1999a] showed that the Dunwoody manifolds included branched covers with singularities that were torus knots of a specific type. L. Grasselli and Michele Mulazzani [2001] showed that Dunwoody manifolds are cyclic coverings of lens spaces branched over $(1,1)$-knots. Cavicchioli, Beatrice Ruini, and Spaggiari [Cavicchioli et al. 2001] proved Dunwoody's conjecture that the Dunwoody manifolds are $n$-fold cyclic coverings branched over knots or links. Soo Hwan Kim and Yangkok Kim [2004] determined the Dunwoody parameters explicitly for a family of cyclically presented groups that are the $n$-fold cyclic coverings branched over certain torus knots and certain two-bridge knots. Nurullah Ankaralioglu and Huseyin Aydin [2008] identified certain of the Dunwoody parameters with generalized Sieradski groups.

6E. Two-bridge knots. The first general presentation about the branched cyclic coverings of the two-bridge knots seems to be that of Jerome Minkus [1982]. A very nice presentation appears in [Cavicchioli et al. 1999b], where cyclic presentations are developed that correspond to cyclically symmetric Heegaard decompositions. In that paper, Cavicchioli, Ruini, and Spaggiari showed that the polynomial of the presentation is the Alexander polynomial. They use the very clever and efficient RR-system descriptions of the Heegaard decompositions. They pass from the Heegaard decompositions to face-pairings and determine many of the geometric structures. Mulazzani and Vesnin [2001] exhibited the many ways cyclic branched coverings can be viewed: polyhedral, Heegaard, Dehn surgery, colored graph constructions.

In addition to these very general presentations, there are a number of concrete special cases in the literature [Bleiler and Moriah 1988; Kim et al. 1998; Kim 2000; Kim and Kim 2003; 2004; Jeong 2006; Jeong and Wang 2008; Grasselli and Mulazzani 2009; Telloni 2010].

Significant progress has been made beyond the two-bridge knots. Maclachlan and A. Reid [1997] and Vesnin and A. C. Kim [1998] considered 2-fold branched covers over certain 3-braids. Alexander Mednykh and Vesnin [1995] considered 2-fold branched covers over Turk's head links. 
Alessia Cattabriga and Mulazzani [Mulazzani 2003; Cattabriga and Mulazzani 2003] developed strongly cyclic branched coverings with cyclic presentations over the class of $(1,1)$ knots, which includes all of the two-bridge knots as well as many knots in lens spaces. P. Cristofori, Mulazzani, and Vesnin [Cristofori et al. 2007] described strongly cyclic branched coverings of knots via $(g, 1)$-decompositions. Every knot admits such a description.

\section{Acknowledgements}

We thank the referees of this and an earlier version of this paper for numerous helpful comments. Purcell is partially supported by NSF grant DMS-1252687 and by ARC grant DP160103085.

\section{References}

[Ankaralioglu and Aydin 2008] N. Ankaralioglu and H. Aydin, "Some Dunwoody parameters and cyclic presentations", Gen. Math. 16:2 (2008), 85-93. MR 2439228 Zbl 1240.57001

[Bergeron and Venkatesh 2013] N. Bergeron and A. Venkatesh, "The asymptotic growth of torsion homology for arithmetic groups", J. Inst. Math. Jussieu 12:2 (2013), 391-447. MR 3028790 Zbl 1266.22013

[Bleiler and Moriah 1988] S. A. Bleiler and Y. Moriah, "Heegaard splittings and branched coverings of $B^{3 ",}$ Math. Ann. 281:4 (1988), 531-544. MR 958258 Zbl 0627.57007

[Boyer et al. 2013] S. Boyer, C. M. Gordon, and L. Watson, "On L-spaces and left-orderable fundamental groups", Math. Ann. 356:4 (2013), 1213-1245. MR 3072799 Zbl 1279.57008

[Brock and Dunfield 2015] J. F. Brock and N. M. Dunfield, "Injectivity radii of hyperbolic integer homology 3-spheres”, Geom. Topol. 19:1 (2015), 497-523. MR 3318758 Zbl 1312.57022

[Brunner 1974] A. M. Brunner, "The determination of Fibonacci groups", Bull. Austral. Math. Soc. 11 (1974), 11-14. MR 0349849 Zbl 0282.20025

[Cannon et al. 2000] J. W. Cannon, W. J. Floyd, and W. R. Parry, "Introduction to twisted facepairings", Math. Res. Lett. 7:4 (2000), 477-491. MR 1783626 Zbl 0958.57021

[Cannon et al. 2002] J. W. Cannon, W. J. Floyd, and W. R. Parry, "Twisted face-pairing 3-manifolds", Trans. Amer. Math. Soc. 354:6 (2002), 2369-2397. MR 1885657 Zbl 0986.57015

[Cannon et al. 2003] J. W. Cannon, W. J. Floyd, and W. R. Parry, "Heegaard diagrams and surgery descriptions for twisted face-pairing 3-manifolds", Algebr. Geom. Topol. 3 (2003), 235-285. MR 1997321 Zbl 1025.57026

[Cannon et al. 2009] J. W. Cannon, W. J. Floyd, and W. R. Parry, "Bitwist 3-manifolds", Algebr. Geom. Topol. 9:1 (2009), 187-220. MR 2482073 Zbl 1179.57030

[Cattabriga and Mulazzani 2003] A. Cattabriga and M. Mulazzani, "Strongly-cyclic branched coverings of $(1,1)$-knots and cyclic presentations of groups", Math. Proc. Cambridge Philos. Soc. 135:1 (2003), 137-146. MR 1990837 Zbl 1050.57003

[Cavicchioli and Spaggiari 2006] A. Cavicchioli and F. Spaggiari, "Certain cyclically presented groups with the same polynomial”, Comm. Algebra 34:8 (2006), 2733-2744. MR 2250565 Zbl 1104.20031 
[Cavicchioli et al. 1998] A. Cavicchioli, F. Hegenbarth, and A. C. Kim, "A geometric study of Sieradski groups”, Algebra Colloq. 5:2 (1998), 203-217. MR 1682984 Zbl 0902.57023

[Cavicchioli et al. 1999a] A. Cavicchioli, F. Hegenbarth, and A. C. Kim, "On cyclic branched coverings of torus knots", J. Geom. 64:1-2 (1999), 55-66. MR 1675959 Zbl 0919.57004

[Cavicchioli et al. 1999b] A. Cavicchioli, B. Ruini, and F. Spaggiari, "Cyclic branched coverings of 2-bridge knots”, Rev. Mat. Complut. 12:2 (1999), 383-416. MR 1740466 Zbl 0952.57001

[Cavicchioli et al. 2001] A. Cavicchioli, B. Ruini, and F. Spaggiari, "On a conjecture of M. J. Dunwoody”, Algebra Colloq. 8:2 (2001), 169-218. MR 1838517 Zbl 0994.57004

[Conway 1965] J. H. Conway, "Problems and solutions: advanced problems: 5327", Amer. Math. Monthly 72:8 (1965), 915. MR 1533441

[Cristofori et al. 2007] P. Cristofori, M. Mulazzani, and A. Y. Vesnin, "Strongly-cyclic branched coverings of knots via ( $g, 1)$-decompositions", Acta Math. Hungar. 116:1-2 (2007), 163-176. MR 2341048 Zbl 1164.57001

[Dunfield and Thurston 2006] N. M. Dunfield and W. P. Thurston, "Finite covers of random 3-manifolds", Invent. Math. 166:3 (2006), 457-521. MR 2257389 Zbl 1111.57013

[Dunwoody 1995] M. J. Dunwoody, "Cyclic presentations and 3-manifolds", pp. 47-55 in GroupsKorea '94 (Pusan, 1994), edited by A. C. Kim and D. L. Johnson, De Gruyter, Berlin, 1995. MR 1476948 Zbl 0871.20026

[González-Acuña and Short 1991] F. González-Acuña and H. Short, "Cyclic branched coverings of knots and homology spheres", Rev. Mat. Univ. Complut. Madrid 4:1 (1991), 97-120. MR 1142552 Zbl 0756.57001

[Gordon 1972] C. M. Gordon, "Knots whose branched cyclic coverings have periodic homology", Trans. Amer. Math. Soc. 168 (1972), 357-370. MR 0295327 Zbl 0238.55001

[Gordon and Lidman 2014] C. M. Gordon and T. Lidman, "Taut foliations, left-orderability, and cyclic branched covers”, Acta Math. Vietnam. 39:4 (2014), 599-635. MR 3292587 Zbl 1310.57023

[Grasselli and Mulazzani 2001] L. Grasselli and M. Mulazzani, "Genus one 1-bridge knots and Dunwoody manifolds”, Forum Math. 13:3 (2001), 379-397. MR 1831091 Zbl 0963.57002

[Grasselli and Mulazzani 2009] L. Grasselli and M. Mulazzani, “Многообразия Зейферта и (1, 1)-узлы", Sibirsk. Mat. Zh. 50:1 (2009), 28-39. Translated as "Seifert manifolds and (1, 1)-knots" in Siberian Math. J. 50:1 (2009), 22-31. MR 2502871 Zbl 1224.57004

[Havas et al. 1979] G. Havas, J. S. Richardson, and L. S. Sterling, “The last of the Fibonacci groups", Proc. Roy. Soc. Edinburgh Sect. A 83:3-4 (1979), 199-203. MR 549854 Zbl 0416.20026

[Helling et al. 1998] H. Helling, A. C. Kim, and J. L. Mennicke, "A geometric study of Fibonacci groups”, J. Lie Theory 8:1 (1998), 1-23. MR 1616794 Zbl 0896.20026

[Hilden et al. 1992] H. M. Hilden, M. T. Lozano, and J. M. Montesinos-Amilibia, "On the Borromean orbifolds: geometry and arithmetic", pp. 133-167 in Topology '90 (Columbus, OH, 1990), edited by B. Apanasov et al., Ohio State University Mathematics Research Institute Publications 1, De Gruyter, Berlin, 1992. MR 1184408 Zbl 0787.57001

[Holt 1995] D. F. Holt, "An alternative proof that the Fibonacci group $F(2,9)$ is infinite", Experiment. Math. 4:2 (1995), 97-100. MR 1377412 Zbl 0853.20019

[Hu 2015] Y. Hu, "Left-orderability and cyclic branched coverings", Algebr. Geom. Topol. 15:1 (2015), 399-413. MR 3325741 Zbl 1312.57001

[Jeong 2006] K.-W. Jeong, "On the cyclic branched coverings of the 2-bridge knot $b(17,4)$ ", Algebra Colloq. 13:1 (2006), 173-180. MR 2188485 Zbl 1093.57001 
[Jeong and Wang 2008] K.-W. Jeong and M.-O. Wang, "Notes on more Fibonacci groups", Algebra Colloq. 15:4 (2008), 699-706. MR 2452002 Zbl 1156.57012

[Johnson 1976] D. L. Johnson, Presentations of groups, London Mathematical Society Lecture Note Series 22, Cambridge University Press, 1976. 2nd ed. published in London Mathematical Society Student Texts 15, Cambridge University Press, 1997. MR 0396763 Zbl 0324.20040

[Juhász 2007] A. Juhász, "On a Freiheitssatz for cyclic presentations", Int. J. Algebra Comput. 17:5-6 (2007), 1049-1053. MR 2355683 Zbl 1182.20036

[Kauffman and Lambropoulou 2002] L. H. Kauffman and S. Lambropoulou, "Classifying and applying rational knots and rational tangles", pp. 223-259 in Physical knots: knotting, linking, and folding geometric objects in $\mathbb{R}^{3}$ (Las Vegas, NV, 2001), edited by J. A. Calvo et al., Contemporary Mathematics 304, American Mathematical Society, Providence, RI, 2002. MR 1953344 Zbl 1014.57009

[Kim 2000] Y. Kim, "About some infinite family of 2-bridge knots and 3-manifolds", Int. J. Math. Math. Sci. 24:2 (2000), 95-108. MR 1775047 Zbl 0963.57003

[Kim and Kim 2003] A. C. Kim and Y. Kim, "A polyhedral description of 3-manifolds", pp. 157-162 in Advances in algebra (Hong Kong, 2002), edited by K. P. Shum et al., World Scientific, River Edge, NJ, 2003. MR 2088439 Zbl 1034.57003

[Kim and Kim 2004] S. H. Kim and Y. Kim, "Torus knots and 3-manifolds", J. Knot Theory Ramifications 13:8 (2004), 1103-1119. MR 2108650 Zbl 1065.57002

[Kim et al. 1998] G. Kim, Y. Kim, and A. Y. Vesnin, "The knot 52 and cyclically presented groups", J. Korean Math. Soc. 35:4 (1998), 961-980. MR 1666482 Zbl 0916.57015

[Lambert 2010] L. Lambert, A toolkit for the construction and understanding of 3-manifolds, Ph.D. thesis, Brigham Young University, Provo, UT, 2010, Available at http://scholarsarchive.byu.edu/etd/ 2188. MR 2801765

[Le 2009] T. Le, "Hyperbolic volume, Mahler measure, and homology growth", lecture slides, 2009, Available at http://www.math.columbia.edu/ volconf09/notes/leconf.pdf.

[Maclachlan 1995] C. Maclachlan, "Generalisations of Fibonacci numbers, groups and manifolds", pp. 233-238 in Combinatorial and geometric group theory (Edinburgh, 1993), edited by A. J. Duncan et al., London Mathematical Society Lecture Note Series 204, Cambridge University Press, 1995. MR 1320285 Zbl 0851.20026

[Maclachlan and Reid 1997] C. Maclachlan and A. W. Reid, "Generalised Fibonacci manifolds", Transform. Groups 2:2 (1997), 165-182. MR 1451362 Zbl 0890.57023

[Mednykh and Vesnin 1995] A. D. Mednykh and A. Y. Vesnin, "On the Fibonacci groups, the Turk's head links and hyperbolic 3-manifolds”, pp. 231-239 in Groups - Korea '94 (Pusan, 1994), edited by A. C. Kim and D. L. Johnson, De Gruyter, Berlin, 1995. MR 1476964 Zbl 0871.57001

[Milnor 1975] J. Milnor, "On the 3-dimensional Brieskorn manifolds $M(p, q, r)$ ", pp. 175-225 in Knots, groups, and 3-manifolds: papers dedicated to the memory of R. H. Fox, edited by L. P. Neuwirth, Annals of Mathematics Studies 84, Princeton University Press, 1975. MR 0418127 Zbl 0305.57003

[Minkus 1982] J. Minkus, The branched cyclic coverings of 2 bridge knots and links, Memoirs of the American Mathematical Society 35:255, American Mathematical Society, Providence, RI, 1982. MR 643587 Zbl 0491.57005

[Mulazzani 2003] M. Mulazzani, "Cyclic presentations of groups and cyclic branched coverings of (1,1)-knots”, Bull. Korean Math. Soc. 40:1 (2003), 101-108. MR 1958228 Zbl 1037.57002 
[Mulazzani and Vesnin 2001] M. Mulazzani and A. Y. Vesnin, "The many faces of cyclic branched coverings of 2-bridge knots and links", Atti Sem. Mat. Fis. Univ. Modena 49:supplement (2001), 177-215. MR 1881097 Zbl 1221.57009 arXiv math/0106164

[Nagasato and Yamaguchi 2012] F. Nagasato and Y. Yamaguchi, "On the geometry of the slice of trace-free $S L_{2}(\mathbb{C})$-characters of a knot group”, Math. Ann. 354:3 (2012), 967-1002. MR 2983076 Zbl 1270.57045

[Newman 1990] M. F. Newman, "Proving a group infinite", Arch. Math. (Basel) 54:3 (1990), 209-211. MR 1037607 Zbl 0662.20023

[Petronio and Vesnin 2009] C. Petronio and A. Y. Vesnin, "Two-sided bounds for the complexity of cyclic branched coverings of two-bridge links", Osaka J. Math. 46:4 (2009), 1077-1095. MR 2604922 Zbl 1191.57012

[Riley 1990] R. Riley, "Growth of order of homology of cyclic branched covers of knots", Bull. London Math. Soc. 22:3 (1990), 287-297. MR 1041145 Zbl 0727.57002

[Rolfsen 1976] D. Rolfsen, Knots and links, Mathematics Lecture Series 7, Publish or Perish, Berkeley, CA, 1976. Revised edition by AMS Chelsea, Providence, RI, 2003. MR 0515288 Zbl 0339.55004

[Sieradski 1986] A. J. Sieradski, "Combinatorial squashings, 3-manifolds, and the third homology of groups”, Invent. Math. 84:1 (1986), 121-139. MR 830041 Zbl 0604.57001

[Silver and Williams 2002] D. S. Silver and S. G. Williams, "Mahler measure, links and homology growth”, Topology 41:5 (2002), 979-991. MR 1923995 Zbl 1024.57007

[Sloane and Guy 1991] N. J. A. Sloane and R. K. Guy, “Associated Mersenne numbers”, pp. A001350 in The online encyclopedia of integer sequences, 1991.

[Szczepański and Vesnin 2000] A. Szczepański and A. Y. Vesnin, "On generalized Fibonacci groups with an odd number of generators”, Comm. Algebra 28:2 (2000), 959-965. MR 1736775 Zbl 0951.20023

[Telloni 2010] A. I. Telloni, "Combinatorics of a class of groups with cyclic presentation", Discrete Math. 310:22 (2010), 3072-3079. MR 2684075 Zbl 1231.05293

[Thomas 1991] R. M. Thomas, "On a question of Kim concerning certain group presentations", Bull. Korean Math. Soc. 28:2 (1991), 219-224. MR 1127741 Zbl 0752.20013

[Vesnin and Kim 1998] А. Y. Vesnin and A. C. Kim, “Дробные группы Фибоначчи и многообразия", Sibirsk. Mat. Zh. 39:4 (1998), 765-775. Translated as "Fractional Fibonacci groups and manifolds" in Siberian Math. J. 39:4 (1998), 655-664. MR 1654144 Zbl 0917.20032

[Vesnin and Mednykh 1996] A. Y. Vesnin and A. D. Mednykh, "Многообразия Фибоначчи как двулистные накрытия над трехмерной сферой и гипотеза Мейергофа-Ноймана", Sibirsk. Mat. Zh. 37:3 (1996), 534-542. Translated as "Fibonacci manifolds as two-fold coverings of the three-dimensional sphere and the Meyerhoff-Neumann conjecture" in Siberian Math. J. 37:3 (1996), 461-467. MR 1434698 Zbl 0882.57011

Received May 26, 2015. Revised January 28, 2016.

JAMES W. CANNON

DEPARTMENT OF MATHEMATICS

BRIGHAM YOUNG UNIVERSITY

279 TMCB

PROVO, UT 84602

UNITED STATES

jmcnnn@gmail.com 
WILLIAM J. FLOYD

DEPARTMENT OF MATHEMATiCS

VIRGINIA TECH

BLACKSBURG, VA 24061

UNITED STATES

floyd@math.vt.edu

LEER LAMBERT

DEPARTMENT OF MATHEMATICS

BRIGHAM YOUNG UNIVERSITY

PROVO, UT 84602

UNITED STATES

leer.lambert@gmail.com

WALTER R. PARRY

DEPARTMENT OF MATHEMATICS

EASTERN MICHIGAN UNIVERSITY

YPSILANTI, MI 48197

UNITED STATES

wparry@emich.edu

JESSICA S. PURCELL

SCHOOL OF Mathematical SCIENCES

MONASH UNIVERSITY

9 RAINFOREST WALK, ROOM 401

Clayton, ViC 3800

Australia

jessica.purcell@monash.edu 


\title{
PACIFIC JOURNAL OF MATHEMATICS
}

Founded in 1951 by E. F. Beckenbach (1906-1982) and F. Wolf (1904-1989)

$$
\text { msp.org/pjm }
$$

\section{EDITORS}

\author{
Don Blasius (Managing Editor) \\ Department of Mathematics \\ University of California \\ Los Angeles, CA 90095-1555 \\ blasius@math.ucla.edu
}

\author{
Paul Balmer \\ Department of Mathematics \\ University of California \\ Los Angeles, CA 90095-1555 \\ balmer@math.ucla.edu \\ Robert Finn \\ Department of Mathematics \\ Stanford University \\ Stanford, CA 94305-2125 \\ finn@math.stanford.edu \\ Sorin Popa \\ Department of Mathematics \\ University of California \\ Los Angeles, CA 90095-1555 \\ popa@math.ucla.edu
}

\author{
Vyjayanthi Chari \\ Department of Mathematics \\ University of California \\ Riverside, CA 92521-0135 \\ chari@math.ucr.edu \\ Kefeng Liu \\ Department of Mathematics \\ University of California \\ Los Angeles, CA 90095-1555 \\ liu@math.ucla.edu \\ Igor Pak \\ Department of Mathematics \\ University of California \\ Los Angeles, CA 90095-1555 \\ pak.pjm@gmail.com \\ Paul Yang \\ Department of Mathematics \\ Princeton University \\ Princeton NJ 08544-1000 \\ yang@math.princeton.edu
}

\section{PRODUCTION}

Silvio Levy, Scientific Editor, production@msp.org

\section{SUPPORTING INSTITUTIONS}

ACADEMIA SINICA, TAIPEI

CALIFORNIA INST. OF TECHNOLOGY

STANFORD UNIVERSITY

UNIV. OF BRITISH COLUMBIA

UNIV. OF CALIFORNIA, BERKELEY

UNIV. OF CALIFORNIA, DAVIS

UNIV. OF CALIFORNIA, LOS ANGELES

UNIV. OF CALIFORNIA, RIVERSIDE

UNIV. OF CALIFORNIA, SAN DIEGO

UNIV. OF CALIF., SANTA BARBARA
KEIO UNIVERSITY

MATH. SCIENCES RESEARCH INSTITUTE

NEW MEXICO STATE UNIV.

OREGON STATE UNIV.
Daryl Cooper

Department of Mathematics

University of California

Santa Barbara, CA 93106-3080 cooper@math.ucsb.edu

Jiang-Hua Lu

Department of Mathematics

The University of Hong Kong

Pokfulam Rd., Hong Kong

jhlu@maths.hku.hk

$$
\text { Jie Qing }
$$

Department of Mathematics

University of California

Santa Cruz, CA 95064

qing@ cats.ucsc.edu

\author{
UNIV. OF CALIF., SANTA CRUZ \\ UNIV. OF MONTANA \\ UNIV. OF OREGON \\ UNIV. OF SOUTHERN CALIFORNIA \\ UNIV. OF UTAH \\ UNIV. OF WASHINGTON \\ WASHINGTON STATE UNIVERSITY
}

These supporting institutions contribute to the cost of publication of this Journal, but they are not owners or publishers and have no responsibility for its contents or policies.

See inside back cover or msp.org/pjm for submission instructions.

The subscription price for 2016 is US $\$ 440 /$ year for the electronic version, and \$600/year for print and electronic.

Subscriptions, requests for back issues and changes of subscriber address should be sent to Pacific Journal of Mathematics, P.O. Box 4163, Berkeley, CA 94704-0163, U.S.A. The Pacific Journal of Mathematics is indexed by Mathematical Reviews, Zentralblatt MATH, PASCAL CNRS Index, Referativnyi Zhurnal, Current Mathematical Publications and Web of Knowledge (Science Citation Index).

The Pacific Journal of Mathematics (ISSN 0030-8730) at the University of California, c/o Department of Mathematics, 798 Evans Hall \#3840, Berkeley, CA 94720-3840, is published twelve times a year. Periodical rate postage paid at Berkeley, CA 94704, and additional mailing offices. POSTMASTER: send address changes to Pacific Journal of Mathematics, P.O. Box 4163, Berkeley, CA 94704-0163.

PJM peer review and production are managed by EditFLOW ${ }^{\circledR}$ from Mathematical Sciences Publishers.

PUBLISHED BY

\section{I. mathematical sciences publishers}

nonprofit scientific publishing

http://msp.org/

(C) 2016 Mathematical Sciences Publishers 


\section{PACIFIC JOURNAL OF MATHEMATICS}

Volume $284 \quad$ No. $1 \quad$ September 2016

Bitwist manifolds and two-bridge knots

JAmes W. CANNON, William J. Floyd, LEeR LAMbert,

WALTER R. PARry and Jessica S. PurCELL

Recognizing right-angled Coxeter groups using involutions

Charles Cunningham, Andy Eisenberg, Adam Piggott and KIM RUANE

On Yamabe-type problems on Riemannian manifolds with boundary

Marco Ghimenti, Anna Maria Micheletti and Angela

PISTOIA

Quantifying separability in virtually special groups

MARK F. HAGEN and PRIYAM PATEL

Conformal designs and minimal conformal weight spaces of vertex operator superalgebras

TOMONORI HASHIKAWA

Coaction functors

S. KALiszewski, Magnus B. LANDSTAD and John QuigG

Cohomology and extensions of braces

VICTORIA LEBED and LEANDRO VENDRAMIN

Noncommutative differentials on Poisson-Lie groups and pre-Lie algebras

SHAHN MAJID and WEN-QING TAO 